\title{
ArcheoSciences
}

Revue d'archéométrie

$29 \mid 2005$

Varia

\section{Dorures des céramiques et tesselles anciennes : technologies et accrochage}

Philippe Colomban, Thomas Calligaro, Claude Vibert-Guigue, Quang Liem Nguyen et Howell G. M. Edwards

\section{OpenEdition}

12 Journals

Édition électronique

URL : https://journals.openedition.org/archeosciences/424

DOI : 10.4000 /archeosciences.424

ISBN : 978-2-7535-1594-9

ISSN : $2104-3728$

Éditeur

Presses universitaires de Rennes

Édition imprimée

Date de publication : 31 décembre 2005

Pagination : 7-20

ISSN : 1960-1360

Référence électronique

Philippe Colomban, Thomas Calligaro, Claude Vibert-Guigue, Quang Liem Nguyen et Howell G. M. Edwards, «Dorures des céramiques et tesselles anciennes : technologies et accrochage »,

ArcheoSciences [En ligne], 29 | 2005, mis en ligne le 31 décembre 2007, consulté le 01 février 2022.

URL : http://journals.openedition.org/archeosciences/424 ; DOI : https://doi.org/10.4000/

archeosciences. 424 


\title{
Dorures des céramiques et tesselles anciennes : technologies et accrochage
}

\author{
Philippe COLOMBAN', Thomas CALLIGARO', Claude VIBERT-GUIGUE, Nguyen Quang LIEM" \\ et Howell G.M. EDWARDS
}

\begin{abstract}
Résumé : Les technologies de dorures des céramiques et verres et leurs évolutions historiques sont très mal documentées. Des échantillons d'époques et de technologies différentes (tesselles d'ćpoque omeyyade, début VIII' s., Qusayr 'Amra, Jordanic ; porcelaine de type « Kinran-de », ćpave de Cu Lao Cham, XV s. Vietnam; porcelaine phosphatique de Rockingham, début XIX's s., Angleterre) ont étć analysés par PIXE et RBS sur l'accélérateur AGLAE et par micro-spectrométrie Raman. Dans tous ces cas, l'or est un alliage $\mathrm{Au}-\mathrm{Ag}$ dont la tencur en argent va de $\sim 5 \%$ en poids pour les deux céramiques à 15\% pour les tesselles. Les épaisseurs des «dorurcs" sont de 3-5 $\mu \mathrm{m}$ pour la céramique vietnamienne et de $1 \mu \mathrm{m}$ pour les autres. Unc couche d'argent, bien accrochéce, cst observée à l'interface alliage $\mathrm{Au}-\mathrm{Ag} / \mathrm{cć}$ ramique ct il est probable qu'elle résulte de la diffusion à la cuisson. De ce travail on peut conclure que la dorure des tesselles du Ville $\mathrm{s}$. a été réaliséc à partir d'une feuille d'or alliée recouverte de verre fondu, que celle de la céramique du XV $\mathrm{X}$, a étć cuite avec les ćmaux et brunie à la pierre dure, alors que la porcelaine du XIX ${ }^{\mathrm{C}} \mathrm{s}$. a étć décoréc à l'or «liquide».
\end{abstract}

\begin{abstract}
To date, the ancient gilding technologies of glasses and ccramics, and their history, are not well documented. In this paper threc examples, Umayyad tesserac (beginning of the $8^{\text {th }}$ century, Qusayr'Amra, Jordan), a "Kinran-de" Vietnamese porcelain (Cu Lao Cham shipwreck, middlc $15^{\text {th }}$ century) and an English Bonc China (Rockingham, beginning $19^{\text {th }}$ century) have been analysed by optical microscopy, $\mu$-Raman scattering, PIXE and RBS (AGLAE). In all cases the gold coating consists of an Au-Ag alloy, $\sim 5 \%$ wt for both the ceramics and $15 \%$ wt for the glass tesserae. A silver film is observed at the interface between the gold film and the silicate substrate, which remains partly when the gilding has been climinated. Film formation is assigned to a segregation at the interface during the firing. It can be conclude that the $8^{\text {th }} \mathrm{c}$. tesserac gold foil has been covered by molten glass, the $15^{\text {th }} \mathrm{c}$. ceramic gold have been fired with the glaze and then burnished and the $19^{\text {th }} \mathrm{c}$. Bone China has been decorated using liquid gold.
\end{abstract}

Mots-clés : Or, céramique, verre, mosaïque, porcelaine, PIXE.

Key-words: Gold, ceramics, glass, tesserae, porcelain, PIXE.

\section{Introduction}

\section{Les données historiques}

Alors que la réalisation d'objets en or remonte aux époques historiques les plus lointaines, l'ornementation de verres et de céramiques par des rehauts d'or n'est largement attestée qu'à partir du XII siècle (Carboni, 2001 ; Ward, 1998) avec les productions de verre et céramiques Fatimides, période faste pour les arts du feu. A cette époque se développa le lustre, une technique de réalisation d'objets imitant l'or pour respecter les préceptes religieux de l'Islam (Caiger-Smith, 1983 ; Colomban, 2004 ; Colomban \& Truong, 2004 ; Perez-Arantegui et al., 2001). Des preuves éparses témoignent cependant que comme pour le lustre les premières productions céramiques rehaussées d'or remontent aux Abbassides. Fernande $z$-Puertas (Fernandez-Puertas, 1998) rapporte la découverte de fragments des époques califales en différents points $d$ 'al-Andalous, datés d'avant le $\mathrm{XI}^{\mathfrak{E}}$ siècle et cite des textes mentionnant leur importation et/ou leur production en al-Andalous dès le début du IX ${ }^{\mathrm{c}}$ siècle.

L'âge d'or des verres dorés culmine aux XIII' et XIV ${ }^{i}$ siècles avec les productions Mamelouk (Egypte et Syrie) et au XIX ${ }^{\mathfrak{c}}$ siècle avec le renouveau pour les arts décoratifs. Les plus anciennes dorures sur verre sont les tesselles des mosaïques. La technique des mosaïques à base de petits cubes de pierre remonte au moins au $\mathrm{III}^{\grave{c}}$ millénaire avant J.-C. avec les productions d'Ur en tesselle de nacre, de lapis lazuli et de schiste. Les Romains

\footnotetext{
'Laboratoire de Dynamique, Interactions et Réactivité (LADIR), UMR 7075 CNRS \& Université P. et M. Curie, 2 rue Henry Dunant, 94320 THIAIS, France. philippe.columban@iglvt-cnrs.fr

${ }^{2}$ Centre de Recherche et de Restauration des Musées de France (C2RMF), Palais du Louvre, 14 quai François Mitterrand, 75001 PARIS, France.

${ }^{3}$ Centre d'étude des peintures murales romaines (CEPMR) UMR 8546, CNRS-ENS, Abbaye Saint-Jean-des-Vignes, 02200 SOISSONS, France.

4 Académie des Sciences et de la Technologie. HANOI, Vietnam.

${ }^{5}$ Chemical and Forensic Sciences, University of Bradford, BRADFORD BD7 IDP, UK.
} 
utilisèrent largement cette technique en sélectionnant différentes couleurs de calcaire où lorsque la palette était insuffisante, en recourrant à de la pâte de verre ou à du verre (Colomban et al., 2003b). L'utilisation de fonds dorés semble être antérieure au $\mathrm{V}^{\mathfrak{k}}$ siècle, certains auteurs (Darque-Ceretti et al., 2002) mentionnant le $I^{\text {er }}$ siècle comme terminus ante quem, sans référence précise à des objets ou lieux. La coupole du mausolée dit de "Galla Placida » (fille de Théodose, empereur d'Orient et régente de l'empire byzantin) édifié à Ravenne de 425 à 430 est à notre connaissance la plus ancienne mosaïque utilisant de larges quantités de tesselles dorées. Une telle maîtrise avec des dizaines de $\mathrm{m}^{2}$ recouverts de tesselles dorées indique une pratique déjà mure. D'autres constructions de Ravenne, un peu plus tardives ( $\mathrm{VI}^{\mathrm{e}}$ siècle), emploient largement les tesselles dorées (Bustacchini, 1973 ; ibidem, 1987). L'utilisation de l'or collö̈dal pour obtenir des verres couleur de sang (Hematinum) est clairement attestée à l'époque romaine comme en témoigne les descriptions techniques de Pline (Barber \& Freestone, 1990 ; Hornyak et al., 1997 ; Bertan, 1913). Le texte de Théophile (milieu du XII ${ }^{\dot{c}}$ siècle) rapporte que les «Grecs» (il faut comprendre les romains) omaient de rehauts d'or des verres bleus en appliquant soit de la feuille d'or, soit un or «liquide» (Withehouse, 1998) et utilisaient de la poudre de verre pour favoriser l'adhésion à la cuisson.

Au Proche-Orient, les tesselles dorées ont rarement été recueillies lors de fouilles et/ou peu étudiées. L'exemple de Qusayr 'Amra a conduit à enquêter sur ce sujet et deux mentions qui concernent des églises, une à Huarte, Syrie (IV-V' siècles) et l'autre à Pétra, Jordanie ont été trouvées (Canivet, 1982 ; Canivet \& Canivet, 1987 ; Fiema et al., 2001). Pour l'époque omeyyade, deux exemples étaient jusque-là connus : le décor intérieur au Dôme du Rocher à Jérusalem, érigé en 692 dans un style byzantin (Grabar, 1997) et la mosquée des Omeyyades à Damas, datant de $\sim 710$ (Mouliérac, 1993). D'autres témoignages commencent à apparaître, mais relatifs à de plus petites surfaces (Khamis, 2001). A Saint-Marc de Venise la plus ancienne mosaïque n'est que du XII' siècle, c'est-à-dire contemporaine de l'essor de l'usage de la dorure sur verre en Islam et à Byzance.

Si les plus anciennes attestations d'or sur céramique semblent être abbassides, les productions largement conservées sont persanes de la fin du XII ${ }^{\grave{c}}$ - début de XIII siècle : «minaï» (céramiques aussi appelées "haftrang » portant un décor multicolore à la manière des enluminures) et Lajjvardina, principalement il-Khanides (céramiques émaillées en bleu au cobalt [Soustiel, 1985 ; Watson, 2004 ; Moulièrac, 1999] et/ou au lapis lazuli [Colomban, 2003c ; Colomban, 2005] de la fin XIII ${ }^{\dot{c}}$ début du XIV ${ }^{\dot{c}}$ siècle). En Asie les premiers décors à rehauts d'or sur céramiques, sur fond bleu, semblent être les productions Yuan (empereurs mongols régnant de 1279 à 1368) (Wang, 2002), la couleur bleue étant la couleur favorite des Mongols (Colomban, 2005). La relation entre les productions Lajjvardina il-Khanides, légèrement antérieures et les productions Yuan est évidente, l'Empire de Kubilai Khan allant de l'Iraq à la Chine via l'Iran et les steppes d'Asie centrale. Ultérieurement les productions Ming des règnes Jiajing (1422-1566) et
Wanli (1573-1620) souvent exportées au Japon sous l'appellation devenue classique de "Kinran-de » utilisèrent largement les rehauts d'or. Ce type de céramiques arriva en Europe par le biais des Compagnies portugaise, hollandaise (VOC), anglaise (East India Compagny) et française (Compagnie des Indes Françaises). Elles servirent alors de modèles pour les productions européennes du XVIII ${ }^{\mathfrak{c}}$ (porcelaines « dures » de Meissen (Reichel \& Schulle, 1984; Sonnemann \& Wächtler, 1984), porcelaines tendres de Saint-Cloud, Mennecy, Chantilly, porcelaines anglaises, etc (Colomban et al., 2004b ; Lahaussois, 1997 ; DallotNaudin \& Jacob, 1983).

Quoique le nombre d'échantillons dans ce travail préliminaire soit très limité, les échantillons choisis, deux tesselles Islamiques du VIII ${ }^{\grave{~}}$ siècle, une porcelaine vietnamienne du milieu du $X V^{\mathfrak{c}}$ siècle et une porcelaine anglaise du début $\mathrm{XIX}{ }^{\mathrm{b}}$ siècle, nous semblent a priori de bons exemples pour une première étude des différentes techniques de dorure. Le premier objectif de ce travail est de formuler des critères susceptibles de différentier les procédés propres à chaque production ou d'attester de pratiques communes. A notre connaissance le seul travail similaire concerne des céramiques modernes (Darque-Ceretti et al., 2002). Des analyses sommaires ont concernées pour des productions de Meissen (Hornig-Sutter, 1985).

\section{Les données techniques}

Les descriptions des techniques anciennes de dorures de céramiques ou de verres sont rares. Le « Manuel Roret» consacré à la «Peinture sur Verre, sur Porcelaine et sur Email », de 1913 résume cependant bien les méthodes utilisées à une période charnière où coexistaient encore méthodes traditionnelles et premiers développements de la chimie moderne. Récemment Landgraf a repris partiellement la description de certaines de ces techniques (Landgraf, 1999).

Pour le verre, le «Manuel Roret» mentionne à la fois la dorure à la feuille, collée à l'huile de lin et au blanc d'cuf, et la dorure chimique, avec ou sans cuisson, la fixation dans le demier cas étant obtenue par du borax ou du borate de bismuth, des fondants énergiques. Avec les méthodes à froid les dépôts sont bien sûrs fragiles, peu permanents. Citons l'utilisation d'un mélange d'une solution d'or (et de $\mathrm{NaCl}$ dans l'eau régale), de soude et d'éther-alcool à $80^{\circ} \mathrm{C}$. Notons que les techniques d'argenture sont différentes : i) précipitation simple à l'ammoniaque à partir de nitrate d'argent et dépôt sur une plaque de verre recouverte de potée d'étain avec ajouts possibles de cyanures de mercure et de potassium, ii) adjonction d'un ajout de bismuth et de plomb, l'opération devant alors être faite à une température permettant la fusion des 3 métaux ( $\mathrm{Pb}, \mathrm{Sn}, \mathrm{Bi})$. D'après certains auteurs (DarqueCeretti et al., 2002 ; Bustachini, 1973) à partir du XV' siècle la couche de verre devant couvrir la feuille d'or était versée en fusion sur la feuille puis l'ensemble était recuit, technique encore utilisée par les artisans italiens.

Pour la réalisation d'un décor d'or sur porcelaine, la poudre d'or est «broyée avec du nitrate de bismuth» (en fait, plutôt du sous-nitrate $\mathrm{BiONO}_{3} \mathrm{H}_{2} \mathrm{O}$ ), du borax étant généralement ajouté pour les décors sur porcelaine dure 
tandis que le borate de plomb est utilisé pour ceux sur porcelaines tendres. La matière est ensuite appliquée à l'aide d'un médium ou vernis (asphalte, huile de lin, essence de térébenthine, gomme, sirop de sucre, miel, etc). A près cuisson au moufle "au rouge cerise ou plus" $\left(\sim 700^{\circ} \mathrm{C}\right)$, l'or doit être rendu brillant par brunissage à l'agate ou à Vhématite. Lior est préparé par dissolution à l'eau régale $\left(3,5 \mathrm{vol} \mathrm{HCl}+1,5 \mathrm{vol} \mathrm{HNO}_{3}\right)$ et précipitation soit au sulfate de fer, soit à la solution de mercure dans $\mathrm{HNO}_{3}$.

Bertan mentionne dans le Mamul Roret une ancienne méthode utilisée à la Manufacture de Sèvres pour les porcelaines tendres, l'or en coquille au miel, recette achetée en 1748 au frère Hippolyte LeFort (d'Albis, 2003); le nom vient du fait que cet or était vendu dans des coquilles de moule. Des feuilles d'or battu sont triturées sur plaque avec du miel pour obtenir une bouillie que l'on dilue à l'eau chaude pour éliminer les grosses particules par décantation; la poudre la plus fine est ensuite mise à sédimenter dans les coquilles de moules. Un mélange obtenu par action du vinaigre sur un mélange d'ail et d'oignon est utilisé comme médium. I a cuisson a lieu vers $750^{\circ} \mathrm{C}$ pour les pâtes tendres et $840-920^{\circ} \mathrm{C}$ pour les pâtes dures. Notons que d'Albis mentionne l'utilisation au XVIII" siècle (p. 267, d'Albis, 2003) du silicate de plomb en remplacement du sel de bismuth. Typiquement les proportions de fondant vont de 5 à $15 \%$ en poids.

Deux remarques au détour du texte de Bertan sont à noter p. 356, "l'or utilisé à Sèves devait être pur ou au plus quelques millicmes d'argent pour donner une dorure éclatante " et p. 366 "l'argen s'applicune fort bien sur les fonds d'émaux colorés, mais alors on $n$ 'y met point de fondant. On applique aussi facilement l'or sur l'argent ".

L'or est aussi utilisé en céramique pour donner le "pourpre de Cassins", appelé aussi " rubis de Kunkel" pour les verres, technique déjà utilisée par les Romains pour préparer les verres rubis Hematinum. Dans le «pourpre de Cusvius» les anciennes recettes de la Manufacture de Sèvres rapportent l'adjonction d'argent, au fondant, pour magnifier la couleur. Les verres rubis sont eux obtenus à partir du chlorure d'or. composé qui est aussi à la base de l'" or hrillant» et de l" or mat". Cette technique fut (re-?) inventée par Kuhn chimiste à Meissen en 1836 : c'est un mélange d'huiles, d'essence de lavande, de térébenthine dans lequel on dissout du soufre à chaud. On peut alors dissoudre $14 \%$ d'or (en poids) à partir du chlorure, les liaisons Au-S, très stables, le permettant, et on obtient un liquide noir se peignant bien. Après ajout du fondant et cuisson l'or obtenu est mince, mais naturellement brillant. Si l'on ajoute jusqu'à $32 \%$ d'or en poudre (d'Albis, 2003), on obtient un or mat qui doit être bruni à la pierre. Un dernier type d'or, "l'or chinois", est obtenu à partir du cyanure AuCN.

Il est clair que les méthodes de dorure sont diverses : la présence d'un " fondant » pour solidariser les particules d'or semble générale (silicate de plomb, sel de bismuth, oxyde de Bore ?). La présence d'autres éléments ( $\mathrm{Ag}$. $\mathrm{Cu}$, etc.) pourrait être une signature de technologie. Nous tenterons d'obtenir ces signatures.

Rappelons aussi la technique décrite par Théophyle ( $c f$. plus haut) de la feuille d'or prise en sandwich entre le support de verre et une couche appliquée en fusion tech- nique qui d'après D.B. Harden cité par D. Whitehouse (Whitehouse, 1998) aurait été utilisée au IV ${ }^{e}$ siècle par les Romains.

\section{Les échantillons}

Nous avons choisi des échantillons donc l'observation visuelle laissait pressentir des technologies différentes (fig. 1).

Tesselles de verre dorées d'époque omeyyade (Qusayr 'Amra, Jordanie)

A $80 \mathrm{~km}$ à l'est d'Amman, le bain de Qusayr 'Amra est réputé pour ses peintures murales du haut Moyen-Age inspirées de l'Antiquité. classées au patrimoine mondial par l'Unesco. A l'époque omeyyade (660-750), princes et califes élevèrent de nombreuses résidences, notamment dans des zones semi-arides que parcouraient nomades et caravaniers. Elles comprennent en général une résidence (qasr), un bain, des structures hydrauliques et une mosquée. l.e décor mural occupe une place importante et dès le XIX' siecle, celui de Qusar 'Amra a retenu l'attention. En effet, bien que d'époque musulmane, il rassemble des tableaux où figurent plus de 250 personnages et autant d'animaux. La représentation du pouvoir, la chasse, les arts, le bain, le zodiaque et bien d'autres thèmes accompagnaient le baigneur ou le visiteur à travers différentes pièces, de la salle d'accueil au caldarium. Du placage de marbres, un peu de stuc et de relief sculpté, des mosaïques au sol complétaient le décor peint. La mission francojordanienne (Institut français d'archéologie du ProcheOrient et Service des Antiquités de Jordanie) chargé du relevé exhaustif des peintures entre 1989 et 1995 a mis en évidence la présence de mosaïques murales dans le caldarium, une pièce célèbre avec sa coupole qui représente l'hémisphère céleste nord (Brunet et al., 1998). Si quelques tesselles de calcaire sont encore en place sur le bandeau qui assure le passage du plan carré au cercle portant la coupole, c'est dans les remblais au sol que furent découvertes des tesselles dorées dans les années 1970 (Almagro et al., 1975). Celles ici sélectionnées proviennent des déblais évacués à l'extérieur du monument, à proximité de l'entrée. Cette richesse picturale associée à une architecture de pierre calcaire toute en harmonie de formes rondes contraste avec l'environnement actuel, steppique semi-aride, traversé par le wadi al-Butum, un épisodique cours d'eau.

Fn Syrie, Qusr al-Hayr al-Gharhi, une imposante résidence dans la steppe à l'ouest de Palmyre, présente également des vestiges de mosaïques murales au-dessus de l'entrée principale. l,a mention archéologique ne décrit pas la nature des tesselles. En revanche, il est signalé du bois sculpté rehaussé de bleu et d'or (Schlumberger. 1986). A l'entrée d'un marché de l'antique Scythopolis, devenue Baysan (Khamis, 2001). un texte en arabe coufique en lettres de tesselles en verre doré sur un fond de cubes bleus est date du règne du calife Hishâm. Dans les grandes cités l'usage des tesselles dorées est attesté sur de vastes compositions murales. C'est le cas à Jérusalem au Dôme du Rocher (Grabar, 1997) et à la mosquée des Omeyyades à Damas (Mouliérac, 1993). 
a)

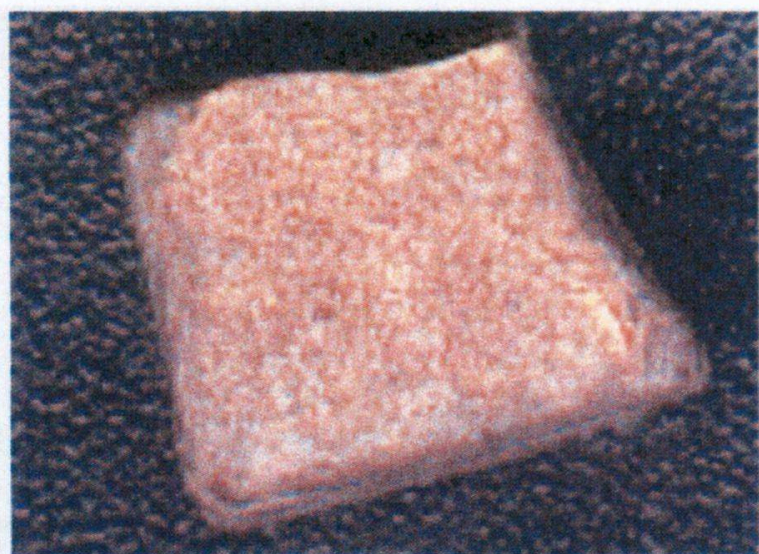

c)

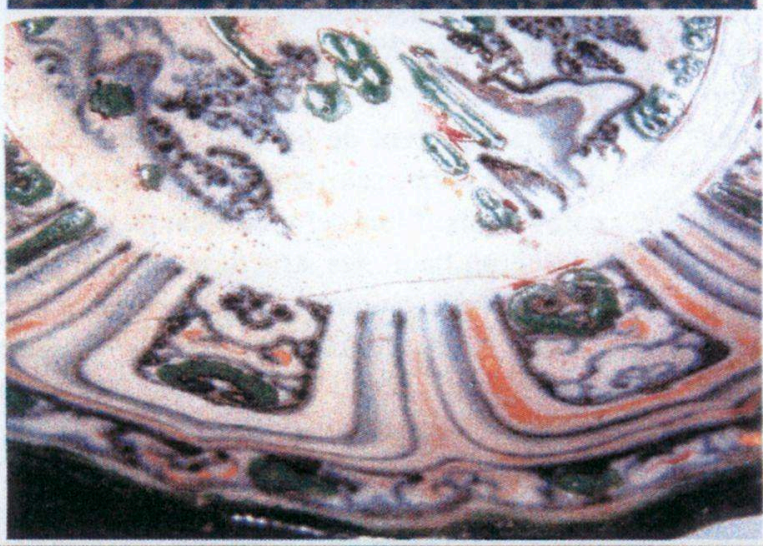

d)

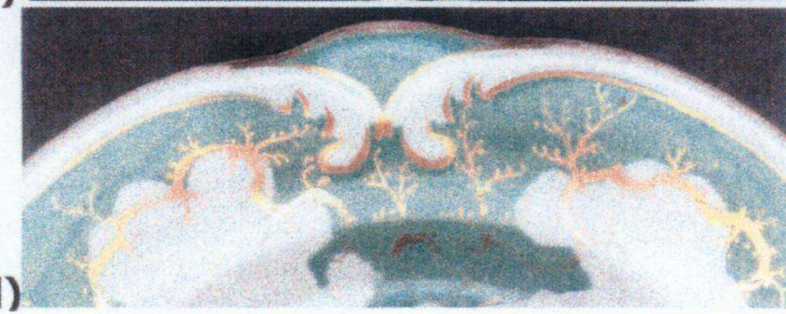

b)
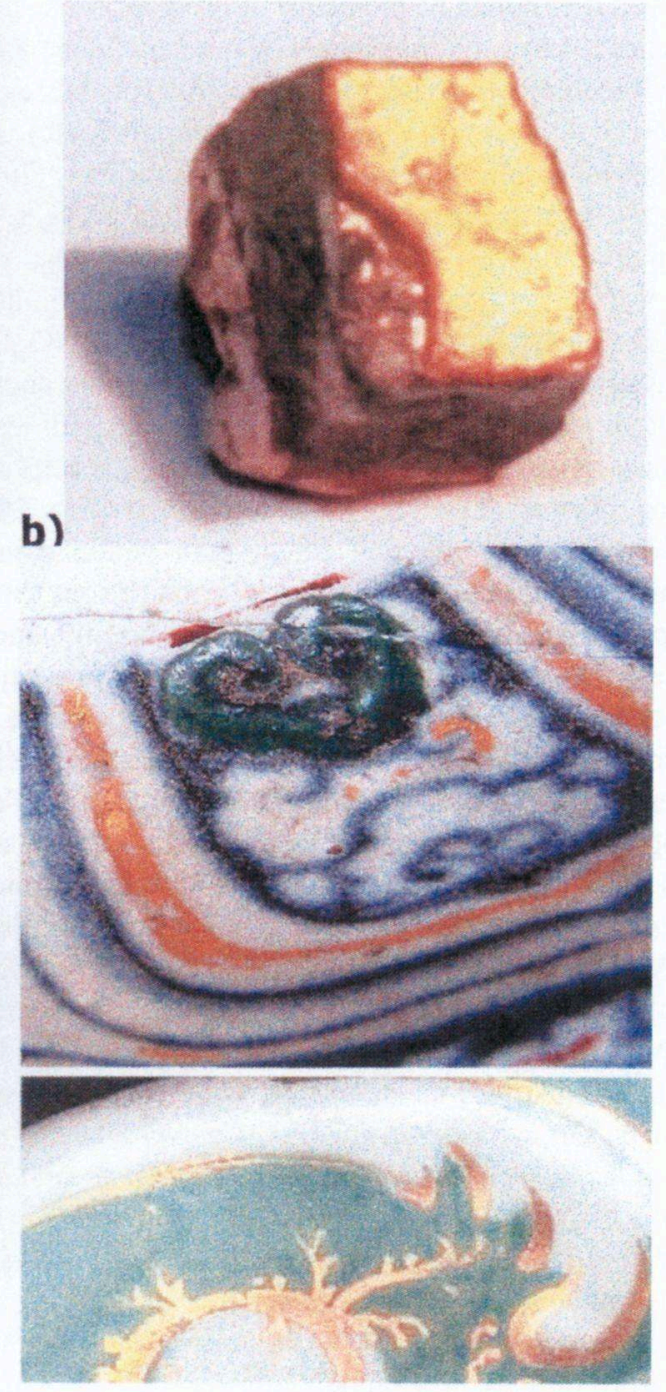

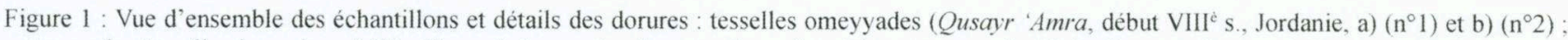
noter sur les tesselles les coins où l'écaillage de la couche de verre de surface laisse apparent le dépôt d'or sous-jacent, c) fragment du plat en grès porcelaineux à décor Kinran-de (Dynastie $L \hat{e}, X V^{e}$ s., Viêt-Nam, épave de $C u$ Lao Cham), photographie du morceau ayant le mieux conservé la dorure ( $c f$. sur la fig. 2 la photo de l'échantillon découpé avec quelques reliquats d'or) et d) assiette en porcelaine phosphatique (Rockingham. XIX $\mathbf{s}$., Angleterre); Photographies (C) Ph. Colomban.

Figure 1: Sample view and detail of the different gilding on Umayyad Qusavr Amra (1 sth $^{\text {th }}$ (ntury, Jordan) \#I (a) \& 2 (b) tesserae; note the out-scaling of the upper glass coating at a corner making the gold layer visible; c) best item, porcelain-like stoneware dish in Kinran-de style (Lê Dynasty, $15^{\text {in }}$ century. Viêt Nam, Cu Lao Cham shipwreck): see fig. 2 the analyzed part; d) Rockingham (Bone China, 19't century, England) porcelain dish; Photograph (C) Ph. Colomban.

A Qusayr 'Amra, les deux tesselles recueillies d'environ $6 \times 6 \times 7$ et $5 \times 6 \times 6 \mathrm{~mm}^{3}$ de volume (fig. 1) sont constituées d'un « cube » de verre épais, lui même comportant 2 couches plus ou moins colorées, sur lequel « une couche d'or » est recouverte par une couche de verre transparent d'environ $0,3 \mathrm{~mm}$. Les bords de cette dernière couche sont écaillés, laissant apparaître quelques $\mathrm{mm}^{2}$ d'or libre (fig. 2) sur lesquels nous pourrons faire les analyses PIXE et RBS.

\section{Céramique bleu-et-blanc rehaussée d'émaux de petit} feu vert et rouge et d'or, Vietnam (XV'è siècle)

Le plat de grès porcelaineux décoré en bleu sous couverte avec rehauts d'émaux vert et rouge et dorure (fig. 1) provient de l'épave ayant coulée au milieu du $X^{\text {è }}$ siècle près d'une l'île située à $20 \mathrm{~km}$ du port de $H o ̂ i$ $A n$ (autrefois appelé $F a i F o$ ) le principal port du Champa puis des royaumes vietnamiens (d'où l'appellation d'île
Cham, «Cu Lao Cham» en vietnamien), escale importante sur la Route des Moussons entre l'Inde et la Chine (Stevenson \& Guy, 1997). Les richesses de l'arrière-pays en faisaient aussi un lieu d'exportation d'ivoire, d'or et de bijoux, de bois, etc. Plus de 240000 pièces céramiques, principalement produites dans le complexe de fours des villages de Chu $\boxminus a ̂ u$ et $M y X a$ (province de Hai Duong, $70 \mathrm{~km}$ Est de Hanoi) furent remontées (Bound, 2002 ; Tri et al., 2002). Quelques très rares pièces et fragments portaient des traces de dorure. A cette période (Dynastie Lê), du fait de désordres en Chine et des décrets impériaux Ming interdisant les exportations, la place occupée par les productions chinoises dans les exportations vers le monde islamique, notamment les Sultanats d'Indonésie, avait été prise par les productions du Tonkin (Stevenson \& Guy, 1997 ; Colomban, 2002a ; ibidem, 2002b). 
Le tesson analysé présente de petits résidus d'or (figs. 1 \& 3). Les plus gros, visibles à l'œil sous forte lumière, ont une surface d'environ $200 \times 200 \mu \mathrm{m}^{2}$ (fig. 3).

\section{Porcelaine phosphatique de Rockhingham (début $X I X^{e}$ siècle, Angleterre)}

La mise au point de la porcelaine phosphatique, un type de porcelaine tendre obtenue à la fin du XVIII ${ }^{\text {è }}$ siècle en utilisant de la cendre d'os (d'où son appellation de «Bone China) ) par Josiah Spode dans le Staffordshire donna naissance à un type de porcelaine d'une remarquable blancheur (Kingery, 1986 ; Kingery \& Smith, 1985). Un des lieux importants de production fut la Manufacture de porcelaine de Rockingham, qui produisit différents services pour la Couronne d'Angleterre. L'assiette analysée (figs. $1 \& 4$ ) porte la marque au griffon indiquant une production entre 1830 et 1842 (Edwards et al., 2004). On peut penser que cette pièce marque soit la fin des techniques traditionnelles, soit le début des méthodes modernes. Le caractère enlevé et le type de dessin des rehauts d'or laissent penser à un dépôt à l'or liquide, au pinceau, voire à un transfert (décalcomanie ou poncif ?)
Le morceau étudié comprenant des filets d'or (largeur 1 à $2 \mathrm{~mm}$ ) posés à cheval sur un émail vert et la couverte transparente ont été analysés en différents points, l'un ayant perdu sa dorure (figs. $1 \& 4$ ).

\section{Méthodes Expérimentales}

\section{Observations au microscope}

Les échantillons ont été observés avec un microscope Olympus muni d'une caméra CCD (grossissement 100 à 1000) associé au spectromètre Raman et avec un microscope digital Hi-scope KH-3000 (Hirox, Tokyo) permettant une mesure précise de la topologie.

\section{Analyse par méthodes PIXE et RBS simultanées avec AGLAE}

Les échantillons ont été caractérisés à l'aide de deux méthodes d'analyse par faisceaux d'ions complémentaires, la méthode PIXE (particle induced X-ray emission / émission $\mathrm{X}$ induite par des particules chargées) et la méthode RBS (Rutherford backscattering spectrometry
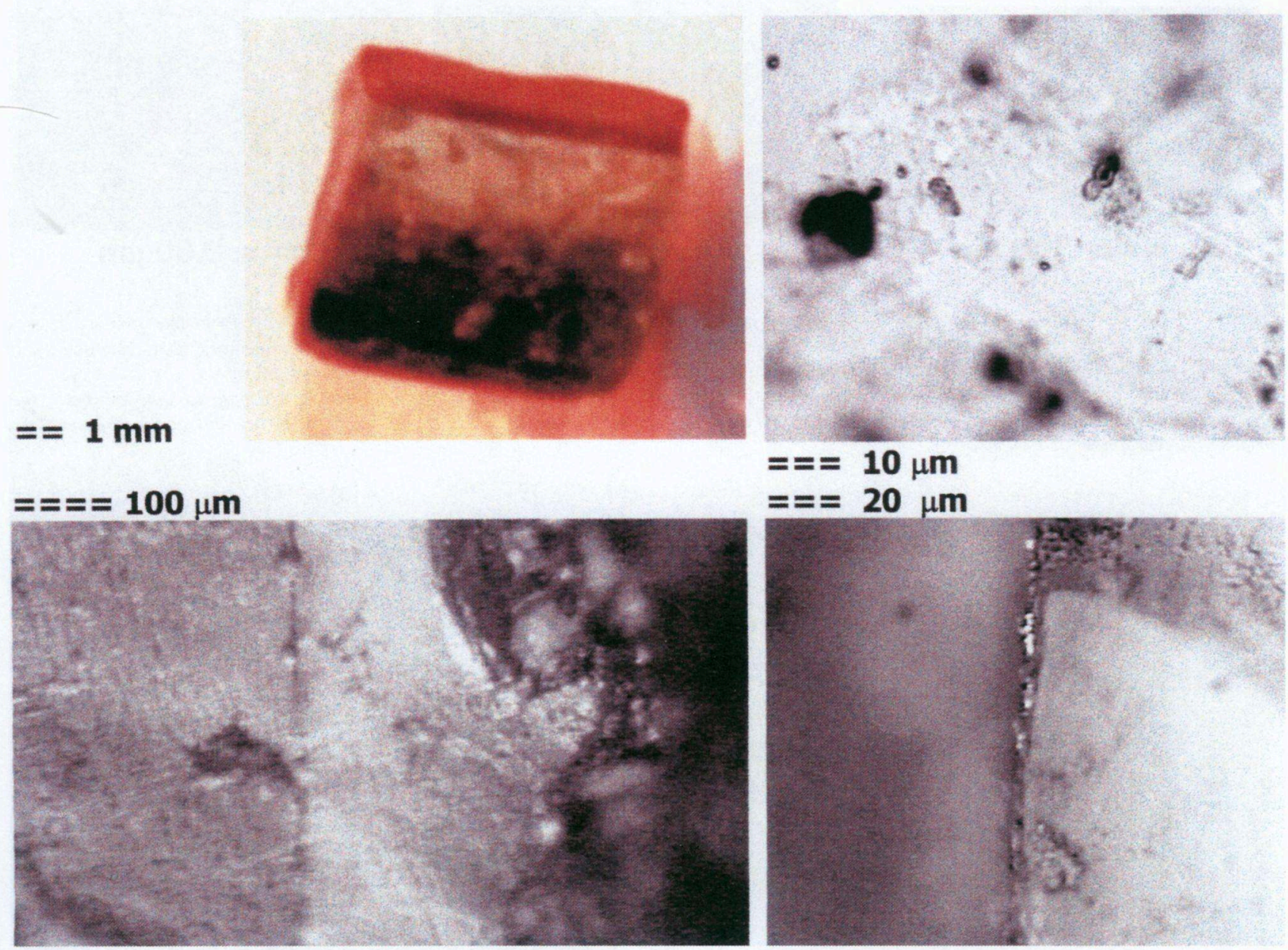

Figure 2: Vue de la tesselle $\mathrm{n}^{\circ} 2$ montrant le verre-support (transparent) et la couche (apparaissant opaque) par dessus le dépôt d'or ; microphotographies montrant une vue de dessus de la surface du dépôt d'or visible dans la zone écaillée (tesselle $\mathrm{n}^{\circ} 1$ ) et l'interface entre le verre support, le dépôt d'or (détail) et à droite, la couche de verre supérieure pour la tesselle $\mathrm{n}^{\circ} 2$; noter les particules brillantes d'or $\left(-3 \times 10\right.$ à $\left.30 \mu \mathrm{m}^{2}\right) ; \mathrm{Photographies} \mathbb{C} \mathrm{Ph}$. Colomban.

Figure 2: \#2 tesserae with its optically clear glass substrate and the upper glass coating on the gold layer (which seems opaque but it also optically clear); optical photomicrographs of the gold layer in a place where the upper coating is away (\#I tesserae) and of the \#2 interface; note the shining gold layer section $\left(-3 x 10\right.$ à $\left.30 \mu m^{2}\right)$; Photograph (C) Ph. Colomban. 

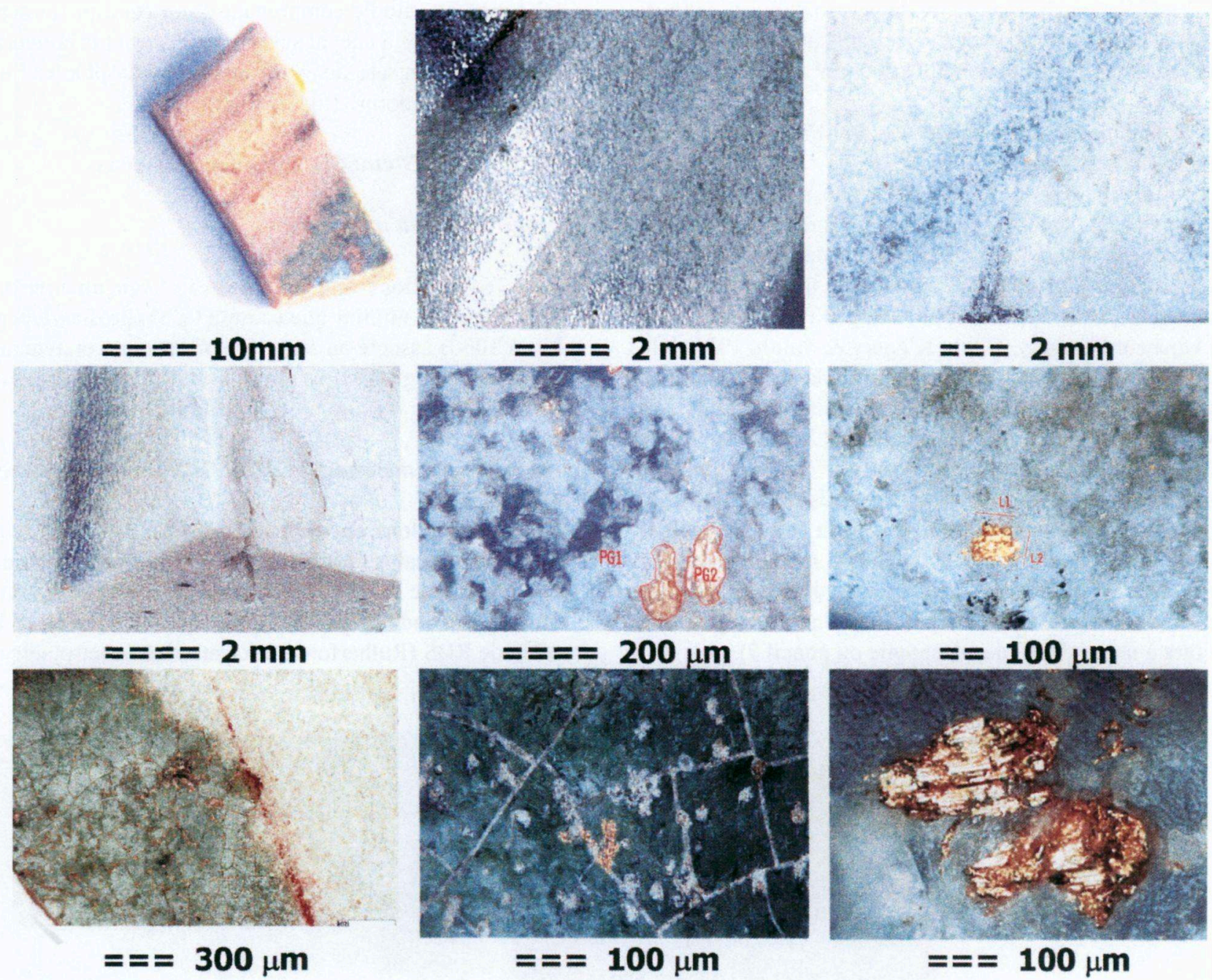

Figure 3 : Détails du plat en grès porcelaineux à décor Kinran-de (Dynastie Lê, $X V^{e} s$., Viêt-Nam) montrant la couche «d'engobe» blanc sur la pâte grise et les bandes mates qui contiennent sur des traînées plus sombres les particules d'or avec leurs stries bien parallèles; quelques très rares reliques d'or sont notées sur l'émail vert. Noter aussi les bandes mates là oủ l'émail rouge a été éliminé ; Photographies (c) Ph. Colomban.

Figure 3: Details of the porcelain-like dish decorated in Kinran-de style (Lê Dynasty, 15 th century, Viêt Nam); see the white glaze on the grey body (section) and the glossy and corrugatedy stripes at the glaze surface with some darker zones; gold particles and hematite are only observed on unglossy stripes : Photograph (C) Ph. Colomban
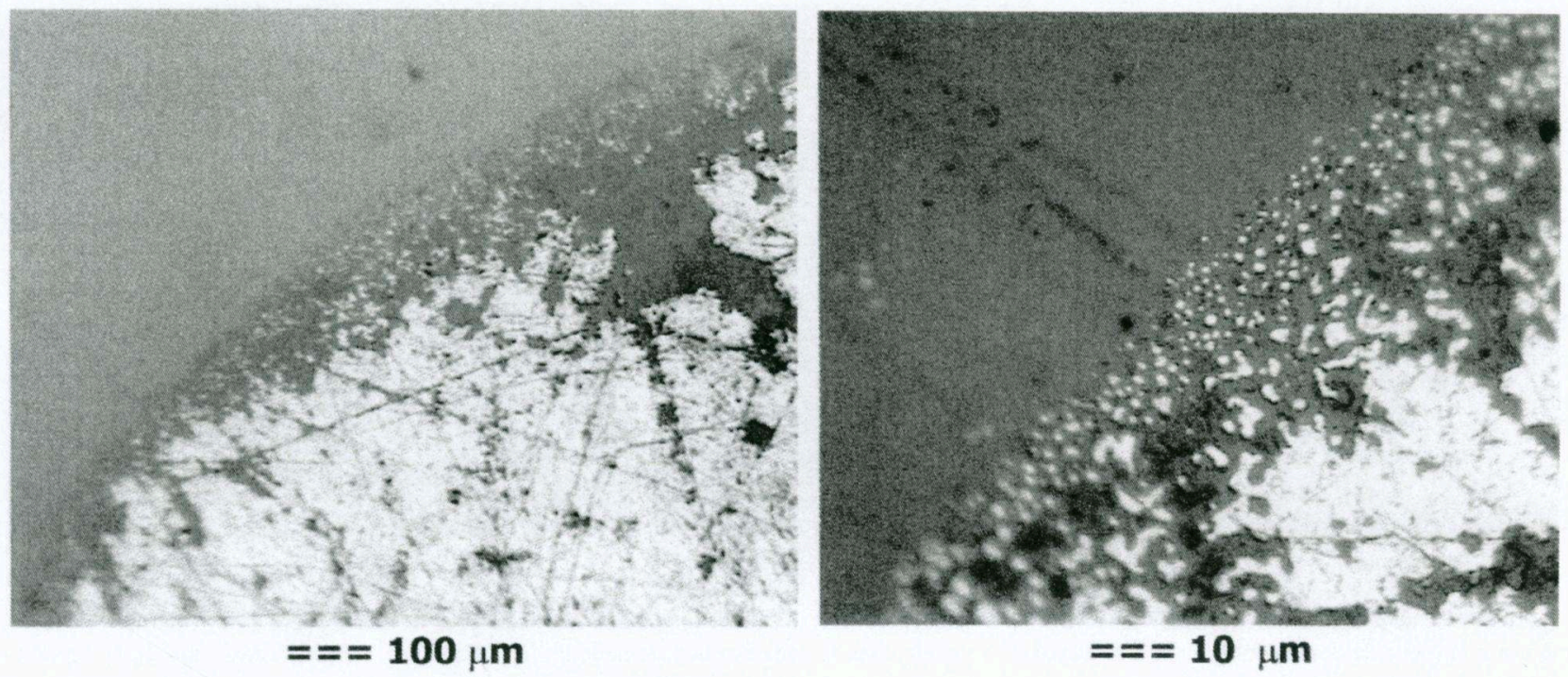

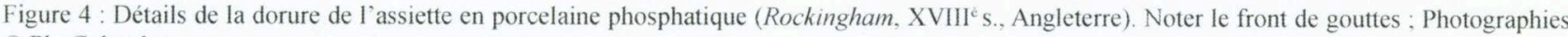
(C) $\mathrm{Ph}$. Colomban.

Figure 4: Optical photomicrographs of the gold décor on Bone China dish (Rockingham, $18^{\text {th }}$ century, England). Note, the front made of many round particles; Photograph (C) Ph. Colomban. 
rétrodiffusion coulombienne). La technique PIXE livre la concentration pour tous les éléments à partir du sodium avec une sensibilité de quelques dizaines de ppm ; elle est bien adaptée à la détermination des compositions d'émaux, verre et corps de céramiques. Elle a aussi été employée pour mesurer les éléments traces dans la «dorure». La technique RBS, qui permet d'estimer les profils de répartition des éléments en profondeur est idéale pour caractériser les dorures (Calligaro et al., 2004). Ces deux techniques sont implantées sur la ligne micro-faisceau/faisceau extrait de AGLAE (Accélérateur Grand Louvre d'Analyse Elémentaire) du Centre de Recherche et de Restauration des Musées de France (C2RMF) qui permet d'analyser les échantillons sans dommages ni préparation, directement «à l'air».

Les échantillons ont été bombardés par un faisceau de protons de $3 \mathrm{MeV}$ avec un courant de $\sim \ln \mathrm{A}$ ). Le diamètre du faisceau au niveau de l'échantillon est de $\sim 30 \mu \mathrm{m}$, une taille bien adaptée à l'analyse des reliquats de dorure conservés sur les deux tessons du Vietnam. Les échantillons sont positionnés vis-à-vis du faisceau à l'aide d'un pointeur laser et d'une micro-caméra. La profondeur analysée est d'une dizaine de microns. La mesure PIXE est effectuée avec deux détecteurs $\mathrm{Si}(\mathrm{Li})$, le premier sous flux d'hélium pour la détection des éléments majeurs, le second derrière un filtre d'aluminium d'une épaisseur de $100 \mu \mathrm{m}$ supprimant les signaux des éléments majeurs pour la détection des traces. La dose intégrée est de $0,5 \mu \mathrm{C}$ sauf pour les essais destinés à rechercher les traces dans l'or où une dose plus forte de $2,5 \mu \mathrm{C}$ a été utilisée. Les temps d'acquisition sont de 3 à 4 minutes, le taux de comptage étant maintenu classiquement sous la limite de 5000 coups/sec. Simultanément les particules rétrodiffusées sont collectées avec un détecteur à barrière de surface placé à $150^{\circ}$ par rapport au faisceau.

Les spectres PIXE ont été traités avec le logiciel GUPIX (Dran et al., 2004) et les spectres RBS avec le logiciel SIMNRA ${ }^{\mathbb{C}}$ 5.0. (Mayer, 2002).

Divers échantillons de référence ont été utilisés pour calibrer les mesures : en RBS, une couche de 1,5 $\mu \mathrm{m}$ d'Au déposée sur du quartz $\left(\mathrm{SiO}_{2}\right)$ et en PIXE, un étalon géochimique diorite « DR-N», un verre au plomb « BGIRA \#3 » et un verre sodique « Brill $B$ ».

\section{Analyse Raman}

Les pièces ont été examinées avec deux spectromètres, un XY (Dilor, Lille, France) dont le premier étage filtrant est un système classique de double monochromateur à réseau, permettant à la fois une très bonne résolution $\left(<1 \mathrm{~cm}^{-1}\right)$ et une mesure des basses fréquences $\left(>30 \mathrm{~cm}^{-1}\right)$ et un INFINITY (Jobin-Yvon-Horiba, Longjumeau, France) beaucoup plus lumineux car il utilise un filtre "Notch », mais qui ne permet pas l'analyse en dessous de $120 \mathrm{~cm}^{-1}$ et dont la précision est plus faible $\left(\sim 2 \mathrm{~cm}^{-1}\right)$. Différents lasers, rouge à 632,82 et $647,1 \mathrm{~nm}$ (avec l'INFINITY et le $\mathrm{XY}$, respectivement) et bleu (487,986 et $457,94 \mathrm{~nm})$ sont utilisés. Les échantillons sont analysés au travers d'un microscope avec des grossissements allant de 100 à 1000 (pour plus de détails voir Colomban et al., 2001 ; Colomban et al., 2003b et Colomban et al., 2004b).

\section{Résultats}

\section{Analyses des supports}

\section{Tesselles de Qusayr 'Amra}

Les signatures Raman du verre support et de la couche recouvrant l'or sont données fig. 5. On observe la signature habituelle d'un verre mixte sodo-calcique (massifs de déformation et d'élongation $\mathrm{Si}$-O culminant respectivement vers $585 \mathrm{~cm}^{-1}$ et $1100 \mathrm{~cm}^{-1}$, tab. 1) avec la présence de pics secondaires bien marqués vers 935 et $1000 \mathrm{~cm}^{-1}$, typiques d'une composition riche en sodium et en calcium (Colomban, 2003c ; Colomban et al., 2003b ; Colomban et al., 2006). Les spectres sont assez semblables mais de petites différences entre les signatures des deux tesselles (et pour une même tesselle entre le verre «support» et la couche supérieure) sont observées. Ces différences de signatures pourraient être utilisées pour chercher dans un corpus plus large des similitudes de technologie ou

\begin{tabular}{|c|c|c|c|}
\hline Echantillon & $\begin{array}{l}\text { Composantes massif } \\
\text { de déformation } \mathrm{Si}-\mathrm{O} \\
\mathrm{cm}^{-1}\end{array}$ & $\begin{array}{l}\text { Composantes massif d'élongation } \\
\qquad \begin{array}{l}\mathrm{Si}-\mathrm{O} \\
\mathrm{cm}^{-1}\end{array}\end{array}$ & $\begin{array}{c}\text { Autres } \\
\text { Signatures } \\
\mathrm{cm}^{-1}\end{array}$ \\
\hline Tesselle QA \#1 & $490,580,635$ & $790,950,990,1025,1090$ & \\
\hline Tesselle QA \#2 & $460,550,585$ & $790,940,1000,1045,1090,1140$ & \\
\hline $\begin{array}{l}\text { Porcelaine CLC } \\
\text { Couverte }\end{array}$ & $350,470,490,570$ & $780,925,985,1050,1140$ & $\begin{array}{l}1330,1600(a) \\
460,510(b)\end{array}$ \\
\hline $\begin{array}{l}\text { Porcelaine CLC } \\
\text { Email vert }\end{array}$ & $475,515,560$ & $760,890,960,1020,1090$ & $\begin{array}{l}635,970(\mathrm{c}) \\
995,1035(\mathrm{~d}) \\
180,195,510,958(\mathrm{e})\end{array}$ \\
\hline $\begin{array}{l}\text { Porcelaine } \\
\text { Rockingham } \\
\text { couverte }\end{array}$ & $405,465,490,585$ & $795,1000,1030,1110,1160$ & $950,1400(\mathrm{~g})$ \\
\hline
\end{tabular}

Tableau 1 : Signatures Raman

Table 1: Characteristic Raman wavenumbers. 
d'origine. Après soustraction des lignes de base suivant notre procédure (Colomban \& Treppoz, 2001 ; Liem et al., 2002 ; Colomban et al., 2006) les spectres ont été décomposés en leurs différentes composantes selon le modèle des $Q_{n}(n=0$ à 4 ) chaque composante correspondant à un type de tétraèdre $\mathrm{SiO}_{4}$, isolé $\left(\mathrm{Q}_{0}\right)$ ou ponté par $1,2,3$ ou 4 liaisons Si-O-Si (Colomban \& Treppoz, 2001 ; Colomban, 2003 a \& b). Les résultats sont résumés dans le tableau 1 et des spectres décomposés sont montrés fig. 5. Les principales variations concernent l'intensité et la largeur du doublet vers $980 \mathrm{~cm}^{-1}$, doublet associé à la saturation en calcium (nanoprécipités de $\mathrm{CaSiO}_{3}$, wollastonite).

Les différentes mesures PIXE sont données dans le tableau 2. Il s'agit bien d'un verre sodo-calcique. On note la présence systématique de soufre $(0,2$ à $0,6 \%$ en poids) et de chlore $(0,7$ à $1 \%)$ comme observé classiquement pour les verres antiques romains obtenus à partir de natron (Henderson, 1985 ; Tite et al., 1998 ; Colomban et $a l ., 2003 \mathrm{~b})$ ainsi que des traces importantes de strontium $(600-800 \mathrm{ppm})$ qui pourrait être un marqueur intéressant de la matière première. On note aussi la présence systématique de manganèse dans la tesselle 2 (1,5 à 3,5\% $\mathrm{MnO})$ que ce soit dans le verre "support» ou la couche «de surface», alors que cet élément est quasiment absent de la première tesselle, ce qui indique une production ou une technique différente. Le verre de la tesselle $n^{\circ} 2$ étant plus transparent et moins coloré, on vérifie le pouvoir «blanchissant» d'un ajout de Mn dans un verre contenant du fer.

En accord avec les petites différences observées en Raman, le verre de la couche de surface est plus riche en

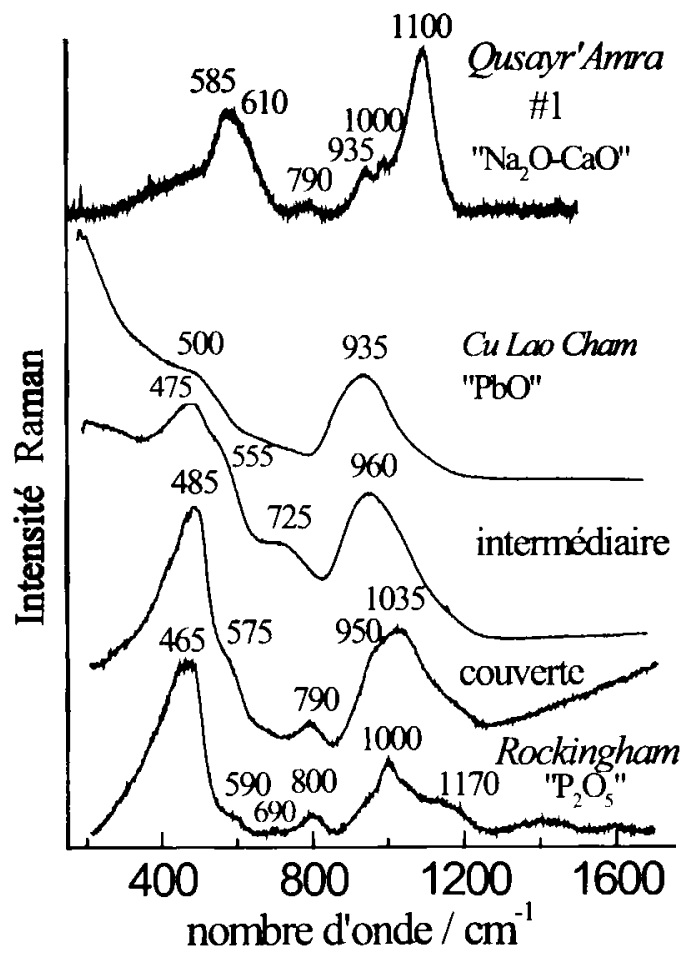

$\mathrm{Na}_{2} \mathrm{O}(10-11 \%$ contre 6-7\% pour le verre support), ce qui doit abaisser son point de fusion et faciliter le nappage.

Grès porcelaineux de l'épave de l'île Cu Lao Cham Comme le montrent bien les photos de la coupe de l'échantillon (fig. 3), sur la pâte grise, dense mais avec des pores allongés dénotant un mauvais désaérage de la pâte du fait de la faible plasticité des pâtes riches en kaolin, apparaît une couche blanche épaisse d'environ $0,3 \mathrm{~mm}$ sur laquelle a été posée le décor, de façon comparable à ce qui est observé sur beaucoup des productions du complexe de fours de Chu $\oplus \hat{a} u$ - $M y X \grave{a}$ (Colomban, 2002b), en accord avec l'origine attribuée à cette cargaison (Tri et al., 2002). La composition de la pâte (tab. 2) est en bon accord avec les précédentes analyses chimiques (Colomban et al., 2003a) : 72 à $76 \%$ (en poids) $\mathrm{SiO}_{2}, \sim 16$ à 22 $\% \mathrm{Al}_{2} \mathrm{O}_{3}$ et $2,5 \% \mathrm{~K}_{2} \mathrm{O}, 0,1$ à $1 \% \mathrm{CaO}$ et $\sim 0,3 \% \mathrm{Na}_{2} \mathrm{O}$; elle correspond bien à un grès porcelaineux donnant une signature de mullite en diffraction $\mathrm{RX}$ et en diffusion Raman. La pâte grise indique une cuisson en réducteur, condition imposée par les teneurs en fer de la pâte et du pigment bleu (tab. 2). La signature Raman de cette pâte est bien celle d'un grès porcelaineux avec la présence de

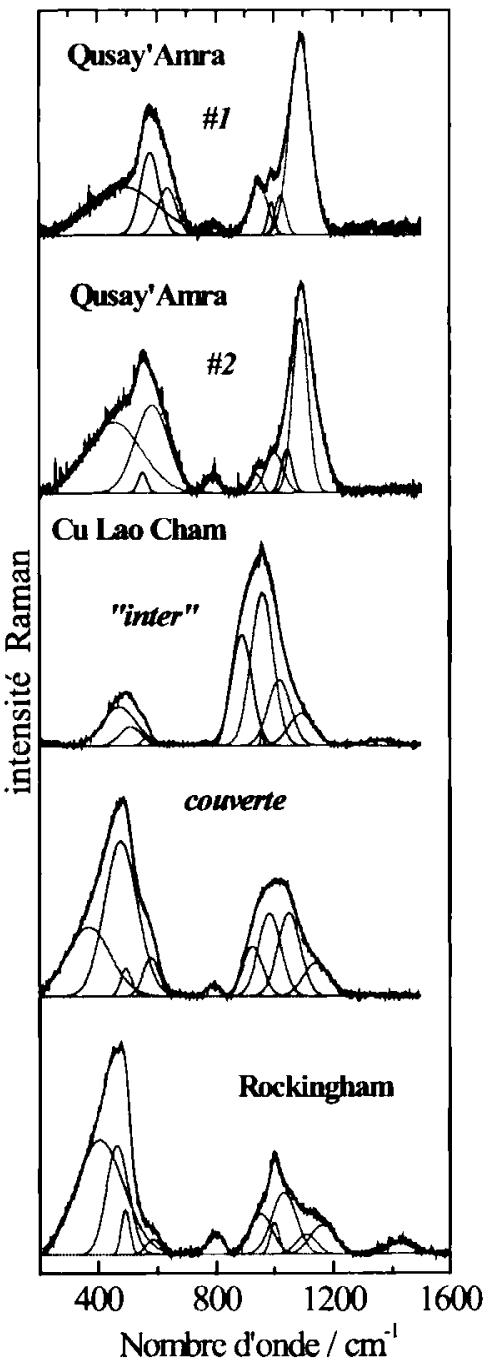

Figure 5 : Spectres Raman représentatifs des différentes phases vitreuses observées dans les différents échantillons. Lcs décompositions cn composantes $Q_{n}$ faites après soustraction de la ligne de base sont indiquées.

Figure 5: Representative Raman spectra for the different glazes/glasses: $Q_{n}$ decompositions are made after base line subtraction. 


\begin{tabular}{|c|c|c|c|c|c|c|c|c|c|c|c|c|c|c|c|c|c|c|}
\hline Echantillon & $\mathrm{SiO}_{2}$ & $\mathrm{Al}_{2} \mathrm{O}_{3}$ & $\mathrm{Fe}_{2} \mathrm{O}_{3}$ & $\mathrm{TiO}_{2}$ & $\mathrm{ZrO}_{2}$ & $\mathrm{MgO}$ & $\mathrm{CuO}$ & $\mathrm{Na}_{2} \mathrm{O}_{2}$ & $\mathrm{~K}_{2} \mathrm{O}$ & $\mathrm{CaO}$ & $\mathrm{SO}_{3}$ & $\mathrm{Cl}^{\circ}$ & $\mathrm{MnO}$ & $\mathrm{P}_{2} \mathrm{O}_{5}$ & $\mathrm{BaO}$ & $\mathrm{PbO}$ & $\mathrm{CoO}$ & $\mathrm{Cr}_{2} \mathrm{O}_{3}$ \\
\hline $\begin{array}{l}\text { Qusayr } \\
\text { 'Amra } \\
\text { surface 2 }\end{array}$ & 73 & 3.5 & 1,1 & 0,2 & & 0,95 & 0,02 & 11 & 1,4 & 9,2 & 0.4 & 0.8 & 3,6 & 0 & 0 & 0,03 & 0,003 & 0 \\
\hline $\begin{array}{l}\text { Qusayr } \\
\text { 'Amra } \\
\text { surface } 1\end{array}$ & 73 & 3,65 & 0,65 & 0,1 & 0,07 & 0,75 & 0,004 & 10,2 & $1 \cdot 1,7$ & 9 & 0,15 & 1,1 & 0,02 & 0 & 0 & 0,005 & 0,003 & 0 \\
\hline $\begin{array}{l}\text { Qusayr } \\
\text { 'Amra } \\
\text { support } 2\end{array}$ & 72,5 & $5-6$ & 1,3 & 0,2 & 0,01 & 0,8 & 0,02 & $3,5-6$ & 2,3 & 7,3 & 0,6 & 3.8 & 3,1 & 0 & $<0,04$ & 0,05 & 0,003 & 0 \\
\hline $\begin{array}{l}\text { Qusayr } \\
\text { 'Amra } \\
\text { support I }\end{array}$ & 75 & 4,7 & 0,7 & 0,1 & 0,01 & 0,9 & 0.01 & 7,6 & 1,2 & 8,5 & 0,2 & ),7 & 0,02 & 0 & 0 & 0,01 & 0,003 & 0 \\
\hline $\begin{array}{l}\text { Porcelaine } \\
\text { Rockingham } \\
\text { páte }\end{array}$ & 38 & 16 & 0,7 & 0,02 & 0,002 & 0,5 & 0,004 & 1,5 & 1,6 & 21,5 & 0 & 0,05 & 0,001 & 20 & 0 & 0,02 & 0,004 & 0 \\
\hline $\begin{array}{l}\text { Porcelaine } \\
\text { Rockingham } \\
\text { couverte }\end{array}$ & 68 & 10 & 0,2 & 0,03 & 0,01 & 1,3 & 0,04 & 1,7 & 3,4 & 8,2 & 1,5 & 0,1 & 0,0006 & 0 & 0 & 9 & 0,004 & 0 \\
\hline $\begin{array}{l}\text { Porcelaine } \\
\text { Rockingham } \\
\text { Email vert* }\end{array}$ & 57 & 5,5 & 0,3 & 0,05 & 0,02 & 0,3 & 0,09 & 4.3 & 2,8 & 9 & 0 & 0,3 & 0 & 0 & 0,09 & 16 & 0,5 & 4 \\
\hline $\begin{array}{l}\text { Porcelaine } \\
\mathrm{Cu} \quad \text { Lao } \\
\text { Cham } \\
\text { pâte }\end{array}$ & 72 & 22 & 1,05 & 0,8 & 0,3 & 0,6 & 0,002 & 0.5 & 2,75 & 0.1 & 0,03 & 0,1 & 0 & 0 & 0,2 & $\sim 0$ & 0 & 0,01 \\
\hline $\begin{array}{l}\text { Cu Lao } \\
\text { Cham } \\
\text { " engobe " }\end{array}$ & 80 & 14 & 0,2 & 0,08 & 0,01 & 0,3 & 0,01 & 0,5 & 2,8 & 2,2 & 0,2 & 0,07 & 0,004 & 0 & 0,04 & 0,03 & 0 & 0,004 \\
\hline $\begin{array}{l}\text { couverte } \\
\text { Cu Lao } \\
\text { Cham } \\
\text { incolore }\end{array}$ & 68 & 13 & 0,6 & 0,2 & 0,06 & 1,4 & 0,06 & 1,4 & 3,2 & 10,6 & 0,4 & 0,5 & 0,1 & 0,2 & 0,2 & 0,005 & 0 & 0,01 \\
\hline $\begin{array}{l}\text { Email } \\
\text { Cu Lao } \\
\text { Cham } \\
\text { vert }\end{array}$ & 28 & 5,4 & 0,9 & 0,1 & 0,05 & 0,65 & 3,7 & 0,01 & 0,5 & 4,2 & 0,7 & 0,6 & 0,03 & 0 & 0 & 53,5 & 0,01 & 0 \\
\hline $\begin{array}{l}\text { Email } \\
\text { Cu Lao } \\
\text { Cham } \\
\text { rouge }\end{array}$ & 65 & 13 & 4,2 & 0,15 & 0,01 & 1,9 & 0,02 & 0,8 & 2,6 & 10,3 & 0 & 0,15 & 0,07 & 0,6 & 0 & 1,3 & 0,06 & 0,008 \\
\hline $\begin{array}{l}\text { Email } \\
\text { Cu Lao } \\
\text { Cham } \\
\text { bleu }\end{array}$ & 76 & 9,9 & 2 & 0,1 & 0,01 & 1,2 & 0,02 & 0,4 & 2,1 & 6 & 0,2 & 0,08 & 0,05 & 0 & 0,04 & 1 & 0,5 & 0 \\
\hline
\end{tabular}

Tableau 2 : Résumé des compositions en \% poids d'oxyde obtenues par analyse PIXE.

Table 2: PIXE compositions (wt\% oxide).

mullite. La signature Raman de « l'engobe » blanc recouvrant le corps (fig. 5), sur lequel est posée le décor en bleu, indique la présence de grains de quartz et de mullite avec des traces de feldspath, sans doute de l'albite (doublet vers 460 et $510 \mathrm{~cm}^{-1}$, Karr, 1975 ; Mernagh, 1991) ainsi que de la wollastonite $\alpha\left(\mathrm{CaSiO}_{3}\right.$, pics fins vers 995 et $1035 \mathrm{~cm}^{-1}$ ) et $\beta\left(635\right.$ et $975 \mathrm{~cm}^{-1}$ ) (Colomban et al., 2004b). Les spectres Raman des zones brillantes, montrent une contamination plus ou moins importante par le plomb de l'émail vert. Les spectres de la fig. 5 montrent deux spectres «limites» l'un saturé en $\mathrm{PbO}$ (avec le faible massif $\delta \mathrm{Si}-\mathrm{O}$ vers $500 \mathrm{~cm}^{-1}$ et un massif $\vee \mathrm{Si}-\mathrm{O}$ culminant à 940-960 $\mathrm{cm}^{-1}$, la corrosion de cet émail donnant un carbonate de plomb, pic fin à $958 \mathrm{~cm}^{-1}$ ) et l'autre de la couverte pure ( $\delta \mathrm{Si}-\mathrm{O}$ vers $485 \mathrm{~cm}^{-1}$ et $v$ Si-O culminant à $\left.1035 \mathrm{~cm}^{-1}\right)$.

L'analyse PIXE montre de fortes concentrations en baryum $(0,2 \%)$ dans la pâte et la couverte, sans doute un marqueur d'une des matières premières, probablement le feldspath.

Le dessin bleu est constitué d'un pigment contenant de l'oxyde de cobalt mais aussi du fer $\left(2 \% \mathrm{Fe}_{2} \mathrm{O}_{3}\right)$ et peut-être du manganèse, comme on l'observe généralement dans les productions vietnamiennes (Colomban et al., 2004c). Aux endroits brillants se trouve une couverte potasso-calcique transparente (tab. 2 ), recouverte d'un émail au plomb $(54 \% \mathrm{PbO})$ coloré en vert $(3,7 \% \mathrm{CuO})$. Une telle teneur en plomb impose une pose sur couverte déjà cuite et une re-cuisson de petit feu. Cette re-cuisson induit une faible contamination de la couverte $(1 \% \mathrm{PbO})$. L'examen de la surface de l'échantillon (fig. 3) montre des zones mates, rugueuses, exemptes d'émail avec des restes, soit d'or, soit d'hématite. Des travaux antérieurs sur les pièces remontées de l'épave ont montré la forte corrosion (y compris par des colonies d'animaux marins) des décors rouges à l'hématite (Colomban, 2002b). Les zones décorées en rouge et à l'or semblent ne pas être protégées par une couche d'émail (absence ou élimination par brunissage ou bien corrosion plus rapide ?). L'émail rouge est riche en silice et en calcium. C'est une espèce de bol d'Arménie, un mélange sable, chaux (?) et hématite. Les « fortes » teneurs en chlore sont attribuées au séjour sous-marin de plusieurs siècles.

Les zones mates où sont observées les paillettes d'or présentent une signature Raman variable et complexe (fig. 6) souvent un mélange de cristobalite $\left(415 \mathrm{~cm}^{-1}\right)$, de quartz $\left(465 \mathrm{~cm}^{-1}\right)$, voire de feldspath $\left(510 \mathrm{~cm}^{-1}\right)$ et peut être de mullite (Colomban \& Treppoz, 2001), avec en de rares endroits la signature d'un verre sodique avec un maximum vers $500 \mathrm{~cm}^{-1}$ et un pic secondaire à $635 \mathrm{~cm}^{-1}$ (fig. 6) comme observé dans les verres fusibles sodiques mis en forme par soufflage (composition de type $75 \%$ 


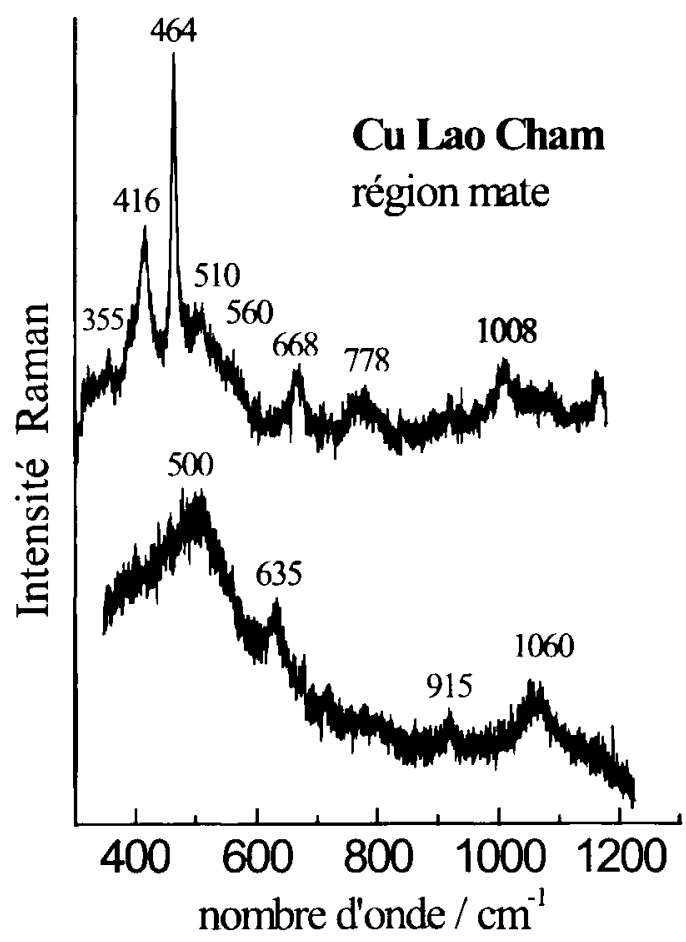

Figure 6: Excmples de spectres Raman mesurés dans les zones mates contenant des paillettes d'or avec signatures caractéristiques de quartz, de cristobalite et de phases non-identifiées avec par endroits des traces de verre.

Figure 6: Raman spectra recorded on the regions with many gold particles with characteristic Raman signature of quartz, cristobalite and unassigned phases and in some places glass traces.

$\mathrm{SiO}_{2} 15 \% \mathrm{Na}_{2} \mathrm{O} 7 \% \mathrm{CaO} 1,5 \% \mathrm{~K}_{2} \mathrm{O}$ ). La très faible teneur en oxyde de fer $(0,2 \%)$ et la forte teneur en silice $(80 \%)$ justifie le qualificatif d'engobe «blanc ». La présence de ce silicate sodique est l'indice que du verre fusible a été utilisé pour « coller » les feuilles d'or.

\section{Porcelaine de Rockingham}

En accord avec les analyses chimiques précédentes (Edwards et al., 2004), la pâte de Rockingham est bien une pâte tendre phosphatique («Bone China»), très riche en phosphate, plus de $20 \%$, comme dans une apatite avec un pic vers $1000 \mathrm{~cm}^{-1}$ et une bande peu intense vers $1400 \mathrm{~cm}^{-1}$ (fig. 5). L'émail vert est plus riche en $\mathrm{PbO}$ que l'émail incolore (respectivement 16 et $9 \%$ ). L'émail vert présente une composition complexe : oxydes d'antimoine, d'étain, de zinc $(1,2 \%)$, de cobalt et de chrome (présence de bleu dans la même zone) ainsi que d'arsenic, ce dernier sans doute associé à la source de cobalt ou de chrome (tab. 2). Son seul fondant est le potassium avec seulement des traces de sodium et de calcium.

\section{Aspect des dorures}

L'examen au microscope des dorures montre des différences importantes. Vue de dessus dans les zones écaillées, l'or des tesselles semble fait de feuilles voire d'un pavement de plaques bien appareillées (fig. 2); est-ce un or à la coquille ou une feuille lissée à la pierre dure ? Sa couleur est plus foncée surtout pour la tesselle $\mathrm{n}^{\circ} 1$ en accord avec la plus forte teneur en argent. L'interface est très nette et l'épaisseur de la " feuille » d'or est, aux endroits les plus épais, de $\sim 1$ à $3 \mu \mathrm{m}$.
L'or de la porcelaine de Rockingham est lisse et brillant (figs. $1 \& 4$ ). Les nombreuses striures sont les traces laissées par les lames de couteaux, cette assiette ayant été utilisée. Par contre à fort grossissement, sur les bords on observe comme des résidus de gouttelettes ce qui laisse penser à un dépôt à «l'or liquide».

Les résidus d'or de la céramique du Viêt-Nam sont principalement présents sur les bandes mates, quelques résidus étant cependant observés sur les zones émaillées en vert comme cela est visible sur les figs. 1 \& 2. De nombreuses stries parallèles sont visibles sur les reliquats d'or de la porcelaine vietnamienne, en accord avec un brunissage à la pierre (fig. 3 ). Sur la surface mate et blanche et sous fort éclairage des traînées noires-bleues sont visibles. Noter la rugosité de la matière, avec des grains de quartz liés par une matière à l'aspect vitreux.

\section{Composition et nature des dorures}

Les résultats sont résumés dans le tableau 2. L'analyse PIXE des dorures Rockingham (porcelaine tendre, début $\mathrm{XIX}^{\mathfrak{c}}$ siècle) et $\mathrm{Cu}$ Lao Cham (grès porcelaineux kaolinitique, milieu $\mathrm{XV}^{\mathrm{c}}$ siècle) donne une composition similaire : $95 \% \mathrm{Au}-5 \% \mathrm{Ag}$ en poids). Par contre les épaisseurs déterminées en traitant les spectres RBS avec le logiciel SIMNRA (Mayer, 2002) (fig. 7) sont différentes : moins de $1 \mu \mathrm{m}$ pour le décor de dorure Rockingham et environ 3 à $6 \mu \mathrm{m}$ pour les différents résidus mesurés sur le tesson Cu Lao Cham. La dorure des tesselles de Qusayr 'Amra a une épaisseur inférieure au micron (fig. 2) mais elle est constituée d'un alliage riche en argent : $85 \% \mathrm{Au}-15 \%$ $\mathrm{Ag}$ en poids. La mesure en certains points d'une quantité importante de silicium pour la dorure $\mathrm{Cu}$ Lao Cham indique des résidus de verre par dessus la dorure ou que la couche de dorure est poreuse. Une oxydation de l'argent n'est pas à exclure car la dissolution importante d'oxygène dans l'argent fondu est un phénomène bien connu (le «rochage»). Une oxydation partielle de l'argent peut jouer un rôle favorable pour l'accrochage substrat siliceux-alliage d'or. La forme des spectres de RBS obtenus sur cet échantillon montre en outre une "large queue" sur le flanc gauche ce qui indique un gradient de concentration. Ceci introduit une incertitude sur la mesure de l'épaisseur des dorures.

Des mesures ont été faites au-delà de la zone d'observation visuelle de l'or. Pour l'échantillon Rockingham, on mesure à côté de la dorure des traces d'or et l'on peut penser qu'il s'agit de résidus de dorure trop petits pour être visibles avec le système de visualisation d'AGLAE. Pour l'échantillon $\mathrm{Cu}$ Lao Cham on mesure par contre un fin dépôt d'argent dont l'épaisseur peut être estimée à $30 \mathrm{~nm}$ (fig. 7). Sous le microscope digital bien adapté pour ce type d'observation (fig. 3) on distingue effectivement des zones noires qui peuvent correspondre aux traces d'un film d'argent oxydé et/ou sulfuré. La difficulté de positionnement du faisceau ne nous a pas permis d'analyser le support de la dorure des tesselles.

Afin de pouvoir analyser finement l'or des échantillons $\mathrm{Cu}$ Lao Cham et de déterminer d'éventuels éléments traceurs caractéristiques d'une source d'or, des mesures PIXE avec une dose plus importante $(2,5 \mu \mathrm{C})$ ont été 
effectuées sur les «paillettes» et juste à côté. Les éléments traceurs généralement recherchés dans l'or sont outre le cuivre et l'étain, les métaux du groupe du platine (Platine, Ruthénium, Rhodium, Palladium, Iridium, Osmium...). La comparaison des spectres (fig. 8) ne montre aucun autre élément que l'or et l'argent dans cette dorure. Notons que Hornig-Sutter (Hornig-Sutter, 1985) rapporte la présence d'un peu d'argent, de bismuth et des
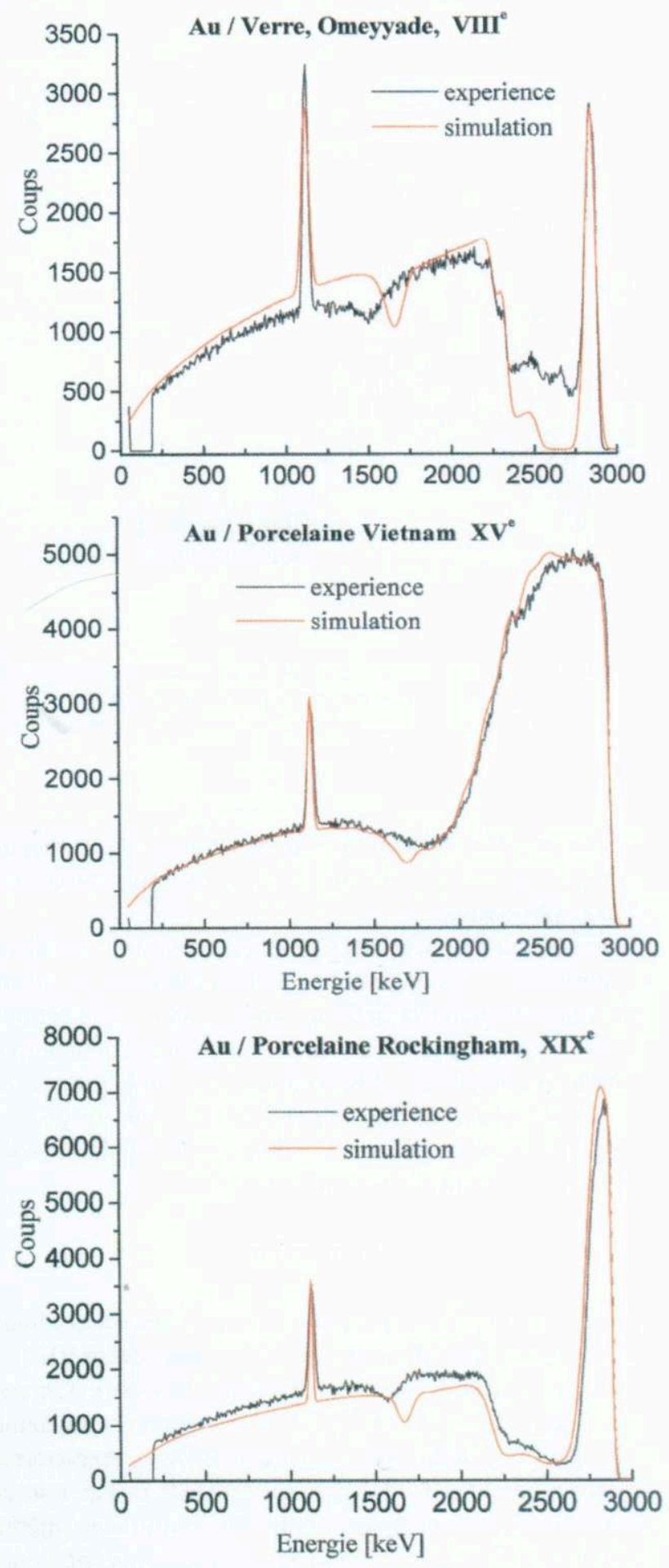

Figure 7 : Comparaison des profils expérimentaux et simulés RBS pour les trois types de dorures; le pic vers $1100 \mathrm{keV}$ résulte du jet d'hélium sur l'échantillon.

Figure 7: RBS experimental profiles and calculations for the different samples (top $8^{\text {th }}$ century, centre $15^{\text {th }}$ century, bottom, $19^{\text {th }}$ century); note the helium $\sim 1100 \mathrm{keV}$ peak. traces de cuivre et de plomb dans l'or de Meissen. Tout au plus on note un peu plus de cuivre à côté que dans la dorure, mais le tesson étant décoré avec un émail vert au cuivre, on peut penser que cette différence provient de la contamination par diffusion lors de la cuisson. L'observation de cuivre associé à l'argent peut aussi être un effet thermique, ces deux éléments diffusant bien dans les verres. Malgré la non-détection d'éléments traceurs, la méthode permet de fixer une limite supérieure à leur concentration : $<300 \mathrm{ppm}$ pour l'étain, $<200 \mathrm{ppm}$ pour l'antimoine. Ces valeurs permettront d'écarter des sources d'or dont la teneur serait supérieure.

\section{Discussion}

\section{Origine et rôle de la sous couche d'argent - Accro- chage Or-Substrat}

Les travaux antérieurs sur de nombreux objets en or ainsi que les données géologiques (Guerra \& Roux, 2004) montrent que l'or est souvent mis en forme à partir d'or natif, un alliage Au-Ag appelé «electrum», avec des teneurs d'argent variant, typiquement entre 3-4 et $20 \%$, rarement plus. L'argent fondant à plus basse température $\left(960^{\circ} \mathrm{C}\right)$ que l'or $\left(1062^{\circ} \mathrm{C}\right)$, il facilite la fusion. Il est donc normal d'utiliser un or plus riche en argent pour un composé moins réfractaire - le verre - et porté à une température a priori inférieure à celle du petit feu des porcelaines. Darque-Ceretti (Darque-Ceretti, 2002) explique l'adhésion de l'or par une diffusion de l'or au travers du substrat. Une comparaison des coefficients de diffusion de l'or $\left(\sim 10^{-17} \mathrm{~cm}^{2} \cdot \mathrm{s}^{-1}\right.$ vers $800-1000^{\circ} \mathrm{C}$ d'après Frischat (Frischat, 1975) et de l'argent $\left(\sim 10^{-5}-10^{-8} \mathrm{~cm}^{2} . \mathrm{s}^{-1}\right)$ dans les verres (Colomban \& Novak, 1992), indique une diffusion plus facile de l'argent, en accord avec la mesure d'un enrichissement en argent dans la zone sous l'or.

Une estimation de l'épaisseur enrichie en argent et en or assimilée à la valeur moyenne $(\mathrm{Dt})^{1 / 2}$ peut être faite pour les différentes conditions de cuisson :

Verre coulé chaud, $\mathrm{t} \sim 3 \mathrm{mn}: \mathrm{Ag} \sim\left(10^{-6} \times 200\right)^{1 / 2} \sim$ $1 \mu \mathrm{m} ; \mathrm{Au} \sim\left(10^{-17} \times 200\right)^{1 / 2} \sim 0$.

Verre recuit, $\sim 4 \mathrm{~h}: \mathrm{Ag} \sim\left(10^{-6} \times 12000^{1 / 2} \sim 10 \mu \mathrm{m} ; \mathrm{Au} \sim\right.$ $\left(10^{-17} \times 12000\right)^{1 / 2} \sim 1 \mathrm{~nm}$.

Cuisson céramique, $\sim 5 \mathrm{~h}: \mathrm{Ag} \sim\left(10^{-6} \times 20000\right)^{1 / 2}$ $\sim 15 \mu \mathrm{m} ; \mathrm{Au} \sim\left(10^{-17} \times 20000\right)^{1 / 2}=$ qlq $\mathrm{nm}$.

Il est donc clair que seul l'argent va pouvoir diffuser significativement dans le substrat (verre ou phase vitreuse des porcelaines) et faciliter un accrochage avec le film d'alliage Au-Ag. Les estimations ci-dessus donnent des épaisseurs de diffusion de l'ordre du micron pour un or chauffé quelques minutes lors du coulage de verre fondu ou d'une dizaine de microns pour les re-cuissons de céramiques. En effet la température de cuisson doit rester inférieure à la température de fusion de l'argent pour éviter les perturbations induites par les problèmes de mouillage. La plus forte teneur en argent de l'or utilisé pour les tesselles peut résulter, soit de l'or utilisé, soit d'une raison technique, la plus faible température de fusion du support. Cela expliquerait aussi la faible largeur du pic RBS, indiquant une répartition en profondeur limitée ; mais outre la plus faible température, le caractère intrinsèquement plus lisse d'un 

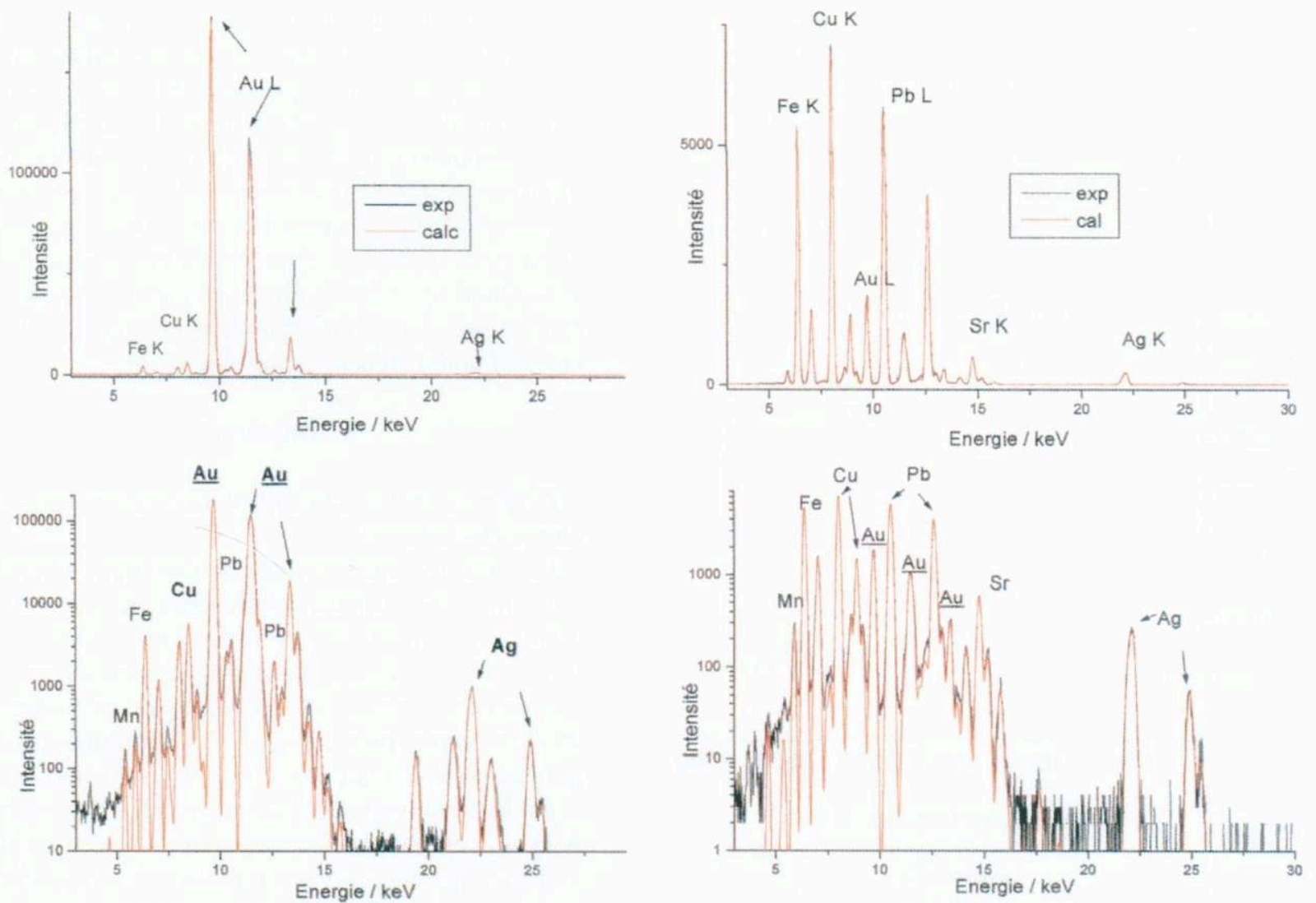

Figure 8: Spectres PIXE des composants majeurs et des éléments traces d'une particule de dorure (gauche) et à côté de cette particule (droite) pour l'échantillon Kinran-de (épave Cu Lao Cham, Dynastie Lê. XV's., Viêt-Nam) ; noter dans le second cas les importants pics d'argent et de cuivre.

Figure 8: PIXE spectra of main constituents and trace elements measured on (left) and out the gold particle (right) on Kinran-de style Lê Dynasty dish (Viet $\mathrm{Nam}, 15^{\text {th }}$ century); note the relative intensity of $\mathrm{Ag}$ and $\mathrm{Cu}$ peaks.

verre et une très probable technique de dorure par recouvrement à l'aide de verre fondu, refroidi rapidement, limitent les possibilités de développer une «large» zone intermédiaire support-alliage. Au contraire la porosité et la rugosité de «l'engobe» du tesson vietnamien explique la queue à gauche de la bande attribuée à la dorure dans le spectre RBS. Il est alors clair qu'une partie de l'accrochage peut alors être physique, favorisé par le brunissage à la pierre.

L'aspect mat, la signature Raman de cristobalite et de quartz (typique d'une céramique, cuite à assez haute température, fig. 6) des zones où sont observées les paillettes d'or et l'absence de réelle contamination en plomb indiquent que l'or, réalisé à partir de feuilles étant donné la forte épaisseur du dépôt, a été déposé de façon concertée avec la pose des émaux. Dans ce cas, soit l'or a été mis en place sur des zones laissées sans couverte et cuit avec cette dernière, soit il a été posé et cuit avec les émaux de petit feu (vert de cuivre et rouge d'hématite). Les résidus observés sur l'émail vert résultent alors d'un transfert lors de l'opération de lissage. Un ajout d'un verre sodique comme agent de collage expliquerait la formation de cristobalite (favorisée par le sodium), l'observation de la signature Raman de verre sodique et les analyses PIXE (présence d'un excès de sodium, absence de bismuth). Les stries clairement visibles sur les paillettes indiquent un brunissage à la pierre dure, opération «classique» pour redonner après cuisson tout son éclat à l'or. Le caractère rugueux de la surface sous l'or favorise alors un accrochage physique.

Les résultats pour la tesselle sont donc tout à fait en accord avec le coulage de la couche supérieure (d'ou son écaillage facile et la belle interface), selon la technique décrite par Théophyle et rapportée comme romaine par Harden (Whitehouse, 1998) tandis que pour la porcelaine de Rockingham, la faible épaisseur, le faciès en gouttelettes des bords de filet et la faible asymétrie du pic sont bien en accord avec un dépôt «d'or liquide».

\section{Conclusion}

Ces travaux préliminaires concernant des échantillons d'époques différentes (tesselles omeyyade, début VIII ${ }^{\mathrm{e}} \mathrm{S}$., Qusayr 'Amra, Jordanie; porcelaine de type «Kinrande », épave de Cu Lao Cham, XV è s. Vietnam ; porcelaine phosphatique de Rockingham, début XIX'̀ s., Angleterre) analysés par PIXE et RBS sur AGLAE et par microspectrométrie Raman ont montré des similitudes (même teneur d'argent de $5 \%$ pour les dorures sur céramique, teneur de $15 \%$ pour celle sur verre, matériaux chauffés à plus basse température) et nous avons pu argumenter une technologie différente pour les trois types d'échantillons, corroborant les textes anciens. Ceci a permis de formuler des questions sur le rôle de l'argent dans l'or utilisé 
comme facteur d'accrochage et de préciser les possibles corrélations entre nature et réfractarité du support et techniques de dorure. L'étude d'un corpus plus significatif est nécessaire pour mieux répondre à ces questions.

\section{Remerciements}

Les auteurs remercient vivement les Drs M. Aucouturier et J. Salomon (C2RMF) pour la fourniture de références et leurs conseils dans l'exploitation des données ainsi que M. X. Faurel, Chef du Laboratoire de la Manufacture de Sèvres pour ses remarques. Monsieur Ho Xuan Tinh, Directeur du Musée de la Région de Quang Nam (Tam Ky) est vivement remercié pour le prêt des échantillons de l'épave $\mathrm{Cu}$ Lao Cham.

\section{Références}

ALBIS, (d') A., 2003 - Traité de la porcelaine de Sèvres. Editions Faton, Dijon

ALMAGRO, M., CABALLERO, L., ZOZAYA, J., ALMAGRO, A., 1975 - Qusayr “Amra. Residencia y Banos Omeyas en cl desicrto de Jordania. Madrid.

BARBER, D.J., FREESTONE, I.C., 1990 - An investigation of the origin of the colour of the Lycurgus Cup by analytical transmission clectron microscopy. Archaeometry, 32: 33-45.

BERTAN, H., 1913 - Nouveau Manuel Complet de la Peinture sur Verre, sur Porcelaine et sur Email. Encyclopédie-Roret, Mulo L Edition, Paris.

BRUNET, J.-P., NADAL, R., VIBERT-GUIGUE, Cl., 1998 - The fresco of the cupola of Qusayr 'Amra. Centaurus, 40: 97-123.

BOUND, M., 2002 - Aspccts of the Hôi An wrcck: dishes, bottles, statucttes and chronology. Taoci 2: 95-104

BUSTACCHINI, G., 1973 - Ravenna tessearac. Gold Bulletin, 6: 52-60.

BUSTACCHINI, G., 1987 - Ravenne, les mosaïques, les monuments et le milieu. Cartolibreria Salbaroli, Ravenne.

CAIGER-SMITH, A., 1983 - Luster Pottery - Technique, Tradition and Innovation in the Islam and the Western Worlds. London, Faber \& Faber.

CALligaro, T., DRAN, J.-C., SAlOMON, J., 2004 - Ion beam microanalysis in Non-Destructive Microanalysis of Cultural Heritage Materials, ch. 5, K. Janssens \& R.V. (jricken, Eds, Elsevier, XLII, 227-276.

CANIVET, P., 1982 - Pcintures murales et mosaiques d'abside en verrc à Huartc (IV-V' s.). In Rayonnement grec. Hommages à Charles Delvoye, Bruxclles, 313-324.

CANivet, P., CANiVET, M.-Th., 1987 - Huartc. Sanctuaire chréticn d'Apamène (IV-V's.), Bibliothèque archéologique et historique, 122: Annexe III, M.-Th. Canivet \& Chr. Lahanier, Verres, 331-346.

CARBONI, S., 2001 - Glass from Islamic Lands - The Al-Sabah Collection. Kuwait National Muscum, Thames \& Hudson, London.

COlombAN, Ph., 2002a - Essor ct apogéc de la céramique vietnamienne. La Revue de la Céramique et du Verre, Juillet-Aout, 125: $18-22$.

COlOMBan, Ph., 2002b - Catalogue de l'exposition « La Fleur du Pĉcher et l'Oiseau d'Azur », 20 avril - 18 août 2002. Musćc Royal dc Maricmont, Bclgique, ISBN 280460638 4, 73-82.

COlomban, Ph., 2003a - Analyse sans contact des céramiques anciennes par diffusion Raman. L'Actualité Chimique, Févricr, 12-16.

COlomban, Ph., 2003b - Polymerisation Degrec and Raman Identification of Ancient Glasses used for Jewellery, Ceramics Enamels and Mosaics. Journal of Non-Crystalline Solids, 323: 180-187.
COLOMBAN, Ph., 2003c - Lapis Lajuli as unexpected blue pigment in Lâjvardina ceramics. Journal of Raman Spectroscopy, 34: 420-423.

COlombaN, Ph., 2004 - Histoirc ct science du lustrc. La Revue de la Céramique et du Verre, 139: 13-21.

COLOMBAN, Ph., 2005 - Route du Lapis Lazuli, lâjvardina, ćchanges cntre arts de la céramique. du verre ct du livre, Taoci, 4: 145-152.

COLOMBAN, Ph., NOVAK, A., 1992 - Defects, non-stoichiometry and phasc transitions, ch.4. In Proton Conductors, Cambridge University Press, Cambridge, 64.

COLOMBAN, Ph., TREPPOZ, F., 2001 - Identification and Differentiation of ancient and modern European porcelains by Raman macro- and micro-spectroscopy. Journal of Raman Spectroscopy, 32: $93-102$.

COLOMBan, Ph., TRUONG, C., 2004 - Non-destructive Raman study of the glazing technique in luster potteries and faience $\left(9-14^{\text {th }}\right.$ centuries): silver ions, nanoclusters, microstructure and processing. Journal of Raman Spectroscopy, 35: 195-207.

COLOMBAN, Ph., SAGON, G., FAUREL, X., 2001 - Differentiation of antique ceramics from the Raman spectra of their colored glazes and paintings. Journal of Raman Spectroscopy, 32: 351-360.

COlomban, Ph., LIEM, N.Q., SAGON, G., hO, X.T., TANG, B.H., 2003a - Microstructure, composition and processing of $15^{\text {th }}$ century Victnamese porcelains and celadons. Journal of Cultural Heritage, 4: 187-197.

COI OMBAN, Ph., MARCH, G., MAZEROLLES, L., KARMOUS, T., AYED, N., ENNABLI, A., SLIM, H., 2003b - Raman identification of materials used for jewellery and mosaics in Ifriqiya. Journal of Raman Spectroscopy, 34: 205-215.

COlOMBAN, Ph., robert, I., ROCHE, C., SAGON, G., MIIANDE, V., 2004b - Identification des porcelaines "tenders" du $18^{\text {¿me }}$ sic̀cle par spectroscopic Raman : Saint-Cloud, Chantilly, Mennecy et Vincennes/Sc̀vres. Revue d'Archéométrie, 28: 153-167.

COLOMBAN, Ph., SAGON, G., HUY, L.Q., LIEM, N.Q., MAZEROLLES, L., 2004c - Vietnamese (15 th century) blue-and-whitc, Tam Thai and Lustre porcclains/stonewares: Glase composition and decoration techniques. Archaeometry, 46: 125-136.

COlomban, Ph., TOURNiĖ, A., Bell.ot-GURLET, L., 2006 Raman identification of glasses and glazes: a tentative differentiation guide, Journal of Raman Spectroscopy, 37: sous presse.

DALLO'T-NAUDIN, V., JACOB, A., 1983 - Les porcelaines tendres françaises - Rouen, Saint-Cloud, Mennecy, Chantilly, Bourg-laReine, Vincennes. Malgras G.J. Ed., abc/Collection, Paris.

DARQUE-CERETTI, E., HELARY, D., AUCOUTURIER, M. 2002 - An Investigation of Gold/ceramic and gold/glass Interfaces. Gold Bulletin, 35: 118-129.

DRAN, J.-C., SALOMON, J., Calligaro, T., WALTER, P. 2004 - Ion beam analysis of art works: 14 years of use in the Louvre. Nuclear Instruments \& Methods, B 219-220: 7-15.

EDWARDS, H.G.M., COLOMBAN, Ph., BOWDEN, B., 2004 Raman spectroscopic analysis of an English soft-pastc porcelain plaque-mounted table. Journal of Raman Spectroscopy, 35: 656-661.

FERNANDEZ-PUERTAS, A., 1998 - Gilded and enamelled glass in al-Andalous, in Ward R., Gilded and Enamclled Glass from the Middle-East, British Museum Press, 74-77.

FIEMA, Z.T., KANELLOPOULOS, CH., WALISZEWSKI, TH. SCHICK, R., 2001 - The Petra church. American Center of Oriental Research Publication 3: Appendix E: The composition of the glass wall mosaic tesserac, Cesare Fiori, 303-305.

FRISCHAT, G.H., 1975 - Ionic diffusion in ionic glasses. Trans. Tech. Publications, Acdermannsdorf.

GRABAR, O., 1997 - Le Dôme du Rocher, joyau de Jérusalem, photographics de S. Nuseibeh. Paris. 
GUERRA, M.P., ROUX, C., 2004 - L'or de la péninsule ibérique des invasions à la reconquista Circulation monćtairc des Wisigoths aux rois chrćtiens. Revue d'Archéométrie, 26: 219-232.

HENDERSON, J., 1985 - The raw materials in early glass production. Oxford Journal of Archaeology, 4: 267-291.

(von) HORNIG-SUTTER, M., 1985 - Meissener Porzellane des 18 Jahrunderts in Visier von Kunstgeschichte und Naturwissenschaft. Weltkunst, Heft 2: 110-115.

HORNYAK, G.I., PATRISSI, C.J., OBERHAUSER, E.B., MARTIN, C.R., VALMETTE, J.C., LEMAIRE, L., DUTTA, J., HOFMAN, H., 1997 - Effective medium theory characterization of $\mathrm{Au} / \mathrm{Ag}$ nanoalloy-porous alumina compositcs. Nanostructured Materials, 9: 571-574.

KARR, C. Jr., 1975 - Infrared and Raman Spectroscopy of Lunar and Terrestrial Mincrals. Academic Press, New-York.

KHAMIS, E., 2001 - Two wall mosaic inscriptions from the Umayyad market place in Bet-Shcan / Baysan. Bulletin of the School of Oriental and African Studies, 64, 159-176.

KINGERY, W.D., 1986 - The development of European porcelains. In "High Technology Ceramics - Past, Present, and Future; The Nature of Innovation and Change in Ceramic Technology" vol. III, Ceramics and Civilization, Kingery W.D. \& E. Lense Eds, The Amer. Ceramic Soc., Westerville, 153-180.

KINGERY, W.D., SMITH, D., 1985 - The development of European soft-paste (Frit) porcelains. In "Ancient Technology to Modem Science vol. I, Ceramics and Civilization, Kingery D.W. Ed, The Amer. Ceramic Soc., Columbus, OH, 273-292.

LAHAUSSOIS, Chr., 1997 - Porcelaines de Saint-Cloud, RMN-La collection du musće des Arts Décoratifs, Paris.

LANDGRAF, G., 1999 - in Gold Progress in Chemistry, Biochemistry and Technology, Schmidbaur H. Editeur, J. Wilcy and Sons, NewYork, 145-1771.

IIEM, N.Q., THANH, N.T., COLOMBAN, Ph., 2002 - Reliability of Raman micro-spectroscopy in analysing ancient ceramics: the case of ancient Victnamese porcelain and celadon glazes. Journal of Raman Spectroscopy, 33: 287-294.

MAYER, M., 2002 - Ion bcam analysis of rough thin films. Nuclear Instruments \& Methods, B 177: 186-195.

MERNAGH, T.P., 1991 - Use of the Laser Raman microprobe for discrimination amongst feldspar mincrals. Journal of Raman Spectroscopy, 22: $453-457$.
MOULIERAC, J., 1993 - Fragment de mosaïque de la grande mosquéc de Damas. Notice du catalogue Syric, Mémoire et Civilisation, Institut du Monde $\wedge$ rabe, Paris, 406-407.

MOULIERAC, J., 1999 - Céramiques du Monde musulman. Institut du Monde Arabe - Snocch, Ducajut \& Zoon, Gand.

PÉREZ-ARANTEGUI, J., MOLERA, J., LARREA, A., PRADELL, T., VENDRELL-SAZ, M., BORGIA, I., BRUNETTI, B.G., CARIATI, F., FERMO, P., MELLINI, M., SGAMELLOTII, A., VITI, C., 2001 - Luster Pottery from the Thirteenth Century to the Sixteenth Century : a nanostructured Thin metallic Film. Journal of The American Ceramic Society, 84: 442-446.

REICHEL, F. \& SCHULLE, W., 1984 - La porcelaine d'ExtrêmeOrient et les efforts déployés en Europe pour en retrouver le sccret. In Mcissen - La découverte de la Porcelainc Européenne en Saxe, J.F. Böttger, Sonnemann R. \& Wächtler E. (sous la direction de) Pygmalion-Gérard Watelet, Paris, 53-75.

SCHLUMBERGER, D., 1986 - Qasr cl-Heir cl-Gharbi. Bibliothèque archéologique et historique, 71: 14-23.

SONNEMANN, R. \& WÄCHTLER, E. (sous la direction de) 1984 Meissen - La découverte de la Porcelaine Européenne en Saxe, J.F. Böttger. Pygmalion-Gérard Watclet, Paris.

SOUSTIEL, J., 1985 - La céramique Islamique. Office du Livre Editions Vilo, Paris, Fribourg.

STEVENSON, J., GUY, J., 1997 - Victnamesc Ccramics: A Scparate Tradition. Art Media Resources, Chicago.

TITE, M.S., FREESTONE, I., MASON, R.B., 1998 - Lead glazes in antiquity - Methods of production and reasons for use. Archaeometry, 45: 241-260.

TRI, B.M., TIN, T.T., LIEM, N.Q., COLOMBAN, Ph., 2002 - How to Date and Identify the Ancient Vietnamese Porcelains and Celadons excavated from the $\mathrm{Cu}$ Lao Cham (Hôi An) Shipwreck. Taoci, 2: $105-110$.

WANG, Q., 2002 - A Dictionary of Chincse Ceramics. Oriental Art Sun Trec Publishing Ltd, Singapore.

WARD, R., 1998 - Gilded and Enamelled Glass from the Middle-East. British Muscum Press, London.

WATSON, O., 2004 - Ceramics from Islamic Lands - The Al-Sabah Collection, Kuwait National Museum, Thames \& Hudson, London.

WITHEHOUSE, D., 1998 - Byzantine gilded glass. In Ward R., Gilded and Enamelled Glass from the Middle-East, British Muscum Press, 4-7. 